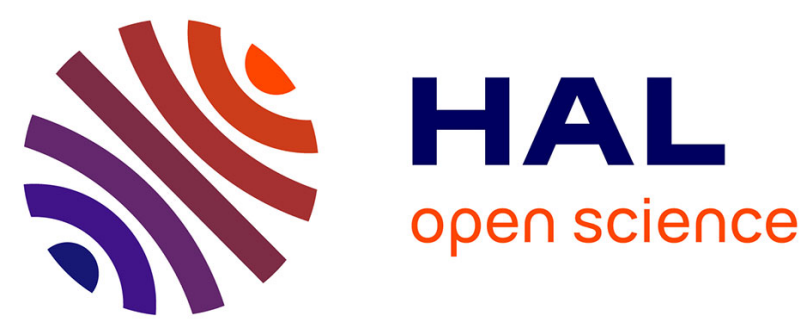

\title{
L'institution des lecteurs
}

Jean-Marie Privat

\section{To cite this version:}

Jean-Marie Privat. L'institution des lecteurs. Pratiques: linguistique, littérature, didactique, 1993, 80, pp.7-34. 10.3406/prati.1993.2355 . hal-03180256

\section{HAL Id: hal-03180256 \\ https://hal.univ-lorraine.fr/hal-03180256}

Submitted on 24 Mar 2021

HAL is a multi-disciplinary open access archive for the deposit and dissemination of scientific research documents, whether they are published or not. The documents may come from teaching and research institutions in France or abroad, or from public or private research centers.
L'archive ouverte pluridisciplinaire HAL, est destinée au dépôt et à la diffusion de documents scientifiques de niveau recherche, publiés ou non, émanant des établissements d'enseignement et de recherche français ou étrangers, des laboratoires publics ou privés. 


\section{L'institution des lecteurs}

Jean-Marie Privat

\section{Citer ce document / Cite this document :}

Privat Jean-Marie. L'institution des lecteurs. In: Pratiques : linguistique, littérature, didactique, ${ }^{\circ}{ }^{\circ 0}$, 1993. pp. 7-34;

doi : https://doi.org/10.3406/prati.1993.2355

https://www.persee.fr/doc/prati_0338-2389_1993_num_80_1_2355

Fichier pdf généré le 13/07/2018 


\section{L'INSTITUTION DES LECTEURS}

\section{Jean-Marie PRIVAT}

"Offrir de lire sans donner tous les moyens de lire vraiment est non seulement inutile mais néfaste " (J.-Cl. Passeron, Le raisonnement sociologique, 1991).

On se rappelle la célèbre circulaire du ministre Rouland (1860) qui instituait la fameuse « armoire-bibliothèque » et déclarait le maître " gardien des livres » (1).

Dans son autobiographie, Edouard Bled se souvient de M. Guillaume, son directeur d'école et du cérémonial hebdomadaire du prêt : "Quand nous désirions des livres, nous l'attendions près de son bureau après quatre heures. II guidait notre choix. La vue de tous ces ouvrages, bien alignés sur leurs rayons, m'émerveillait par leur nombre. J'en aimais l'odeur humaine, l'odeur des livres qui ont passé fraternellement de main en main, de foyer en foyer. Ils étaient tous du plus beau noir » (2). Ce culte républicain du livre de lecture perdurera et des générations de petits lecteurs liront dans Le Tour de la France par deux enfants que les " bibliothèques scolaires sont les bienfaits de notre patrie " et que « M. l'instituteur prête des livres aux écoliers laborieux » (3).

Pendant près de cent ans, mis à part bien entendu, les initiatives originales et les dispositifs précurseurs (bibliothèque pour enfants de L'Heure Joyeuse, à Paris (1924), albums du Père Castor, pédagogie Freinet, coin-bibliothèque, par exemple), telle fut fondamentalement la « présence » du livre, à l'Ecole du moins.

(1) U. ROBERT, «Circulaire relative à l'établissement de bibliothèques scolaires dans les écoles primaires publiques (31 mai 1860) ", Recueil de lois, décrets, ordonnances, arrêtés, circulaires, etc. concernant les bibliothèques publiques, communales, universitaires, scolaires et populaires, Paris, Champion, 1883, pp. 209210

(2) E. BLED, Mes Ecoles, Paris, Laffont, 1977, p. 65.

(3) G. BRUNO, Le Tour de la France par deux enfants, Paris, Belin, 1976 ( $1^{\text {re }}$ édition en 1877), pp. 43-45. 
Aujourd'hui, la situation est radicalement changée, dans l'offre et la demande du livre mais aussi dans les conceptions et les exigences du développement culturel.

Pour illustrer les changements qui ont bouleversé le marché du livre, accru sa présence protéiforme et sa visibilité, il suffit d'évoquer le développement spectaculaire des bibliothèques publiques et des médiathéques municipales, les 30 à 35000 titres publiés chaque année en France dont 10.000 nouveautés, les 10.000 points de vente du livre répartis sur le territoire et la présence du livre dans les grandes surfaces, la multiplication des collections de poche et des clubs de lecture, la montée en puissance des bibliographies minitélisées, des consultations télématiques et des textes numérisés (4), la surabondance de la production de livres ou de revues pour la jeunesse, les incessantes sollicitations médiatiques ou locales pour fêter "furieusement » le livre, etc.

Autrement dit, de l'armoire-bibliothèque d'autrefois à l'offre du livre aujourd'hui, on passe d'un médiateur proche, direct, unique et connu, à un système de médiations où les médiateurs sont nombreux et les médiations polymorphes parce qu'il y a à la fois pluralisation des lieux du livre, prolifération des objets à lire, complexification des réseaux de communication culturelle, sophistication des codes d'accès aux livres, renouvellement rapide des stratégies de traitement de l'information écrite (de la lecture en zapping (5) à la lecture sur écran, de l'interrogation d'une banque de données au CD interactif), diversification des valeurs et des enjeux des lectures, multiplication des sociabilités plus ou moins ritualisées de lecteurs (clubs ou salons du livre, sorties à la bibliothèque, achats de livres-cadeaux à Noël, etc.).

Aussi, l'Ecole ne peut plus se contenter, sous une forme ou sous une autre, d'octroyer solennellement ou prosaïquement des livres ni même continuer à seulement valoriser la lecture sans se préoccuper des moyens techniques et culturels d'accès à une véritable «culture du livre » et plus généralement des écrits. Elle peut d'autant moins se satisfaire d'un discours de la légitimité et d'un apprentissage élémentaire que de nouveaux concepts éducatifs fondamentaux s'imposent peu à peu. Le statut de l'enfant lecteur ou du jeune lecteur évolue vers plus de liberté culturelle, l'attitude à l'égard du savoir est plus constructive et plus exploratoire (et pendant plus longtemps) qu'imitative et récitative, la pédagogie de l'autonomie culturelle se répand (B.C.D., C.D.I.), le libre accès à la documentation est la règle, les exigences scolaires et sociales de maîtrise des écrits croissent. Aussi, un vrai lecteur n'est plus celui qui lit le livre qu'on lui propose (ce modèle républicain est daté) mais celui à qui on apprend à se construire un chemin dans l'univers pratique et symbolique de la culture écrite, d'en saisir les enjeux et l'intérêt, pour lui (modèle libéral et démocratisé). L'Ecole peut aider à développer cette compétence plurielle et fonctionnelle de lecteur, cette culture de lecteur (comme on parle ethnologiquement d'une culture de rocker ou d'une culture de pêcheur).

Avant d'analyser les conditions socio-ethnologiques d'une pratique culturelle

(4) Voir par exemple l'article joyeusement provocateur de M. MELOT, « Nous sommes tous des illettrés ou l'avenir de la lecture ", Bulletin des Bibliothèques de France, t. 34, № 2-3, 1989, pp. 203-207.

(5) Voir par exemple les analyses de J.-F. BARBIER-BOUVET, Lire la page comme une image, Les Entretiens Nathan, III, Paris, 1993, pp. 225-239. 
et de présenter les principales perspectives didactiques qui en découlent, nous voudrions attirer d'abord l'attention sur quelques prises de positions récentes ou à la mode (mais pas forcément nouvelles...) qui paralysent explicitement le travail de médiation voire, ce qui est peut-être plus pernicieux, le détourne de son sens.

\section{LE DOUBLE TRAVAIL DU MÉDIATEUR (6)}

Les exemples qui suivent illustrent les principaux discours théorico-médiatiques ou les pratiques communes inspirés d'un légitimisme culturel latent ou patent qui neutralisent le travail du médiateur sur lui-même (maîtrise de ses propensions culturelles spontanées) et le travail de reconnaissance de la culture de l'autre (ses affinités et ses différences / différends plus ou moins stabilisées avec les pratiques culturelles proposées par l'institution).

\section{Le rapport im-médiat}

Pour certains, il est inutile, nuisible même de travailler à construire des compétences pratiques de lecteur car toute stratégie appuyée de familiarisation fait perdre au livre "son aura de prestige, de grandeur et d'obscurité »; on ne devrait s'approcher du livre que « dans un rapport privé, solitaire, silencieux » parce que toute didactique du livre en fait un " objet profane, laïque, quotidien et commun, [...], une valeur dégradée » (7).

On reconnaît bien dans cette sacralisation du livre, dans cette proposition de rencontre im-médiate avec l'œuvre, l'idéologie de la grâce culturelle et la communion lecturale. Cette liturgie légitimiste tend évidemment à façonner les représentations de nombre de médiateurs au point de rendre improbable toute conversion culturelle organisée et toute initiation systématique à la culture des livres (8).

On retrouverait aisément un écho de cette représentation romantique du lecteur solitaire et inspiré dans le dispositif scolaire traditionnel de la rédaction où les vertus de l'imitation et de l'inspiration constituent l'essentiel des aides à l'écriture.

\section{Le rapport fétichisé}

Ce discours qui tend volontiers à universaliser un rapport privilégié à la culture sans se préoccuper d'en universaliser les conditions techniques et symboliques

(6) Cette partie a été développée une première fois à l'occasion du colloque "Littérature et Jeunesse ", organisé à I'INRP par Ch. POSLANIEC, en mars 1993 (actes à paraître).

(7) D. SALLENAVE, "La lecture - ou le livre? ", Bulletin de l'Association pour la défense et l'illustration de la littérature contemporaine, 3, janvier 1985, pp. 4-6.

(8) Pour un approfondissement de l'analyse critique de cette conception culturellement élitiste et conservatrice, voir par exemple P. BOURDIEU et A. DARBEL, L'Amour de l'art, Paris, Minuit, 1966 et plus récemment, P. BOURDIEU, Les Règles de l'art, Paris, Seuil, 1992. 
d'accès (9) se retrouve dans un autre type de rapport qu'on peut appeler le rapport fétichisé.

Prenons à titre d'exemple l'inquiétant article d'Henri Mitterand paru il y a peu dans la revue Le Débat (10). Pour cet éminent professeur, le salut culturel est dans " la lecture des grandes œuvres, en elles-mêmes et pour elles-mêmes. C'est de là que tout découle, à commencer par l'intérêt que les élèves portent à la classe de français ». "La rencontre avec la littérature et l'art » n'est donc pas pensée comme problématique puisque par son charisme même l'œuvre doit rencontrer « le potentiel de réception d'un esprit libre » (11).

Cette conception charismatique de la littérature et cette conception magique de son appropriation ne nous retiendraient guère si elles n'étaient redoublées (fatalement ?) d'un ethnocentrisme culturel extrême : ceux qui choisissent le loto ou le jeu télévisé plutôt qu'Eschyle ou Giorgione sont des " non-libres » et resteront toute leur vie des espèces de "légumes".

Cette violence symbolique est évidemment peu propice à une prise en compte attentionnée de la culture de l'autre et à une mise en place minutieuse de situations d'apprentissages culturels. Confondre médiateur et grand prêtre c'est s'exposer à ne convertir que les croyants. Plus grave encore, ce " fétichisme culturel » fait que les œuvres, « d'instruments de production, donc d'invention et de liberté possible, sont converties en héritage, routinisé et réifié » (12).

\section{Le rapport privilégié}

Avec Daniel Pennac et son très médiatique essai Comme un roman (13), apparaît un travail de micro-socialisation culturelle fondé moins sur l'objet culturel que sur le médiateur et son charisme personnel cette fois : en racontant chaque soir une histoire nouvelle pendant que son enfant glissait dans les pyjamas du rêve, le narrateur et son petit auditeur formaient, littéralement un seul corps littéraire : "Nous étions le Livre ». Pareillement, en classe, ce qui

(9) D. SALLENAVE, par exemple, raconte en ces termes son enfance lectrice qui baigne dans la religion naturelle du livre (valeurs, imaginaire, pratiques, rites et personnes confondus) : « Nous avons toujours su lire : nous ne pouvons pas nous rappeler le temps où nous ne savions pas lire, une brume d'où émergent des mains, des visages, des faces plates, trouées de sourires et d'yeux et, au-dessus de nos têtes, un mouvement de rideaux. Notre enfance nous semble faite d'après-midis lentes, de soirs tombants dans une salle de classe. Dehors, la nuit se rapprochait des carreaux, et bientôt, en regardant par la fenêtre, nous ne voyions plus que notre propre visage, une rangée de livres, et le rougoiement du poêle [...]. Nous lisions. Le monde commençait tout près, de l'autre côté des vitres froides. Nous lisions.

Les livres n'étaient pas des livres, ils étaient le monde. Les livres avaient été écrits de toute éternité ; ils étaient aussi immuables que le soleil et que l'eau ; ils avaient une existence nécessaire, aussi divine que la leur. Le monde s'accomplissait dans les livres, dans le formidable silence des campagnes et la vacuité des années d'enfance où nous n'avons fait que lire " (Eternités parallèles ", La Revue des Lettres Modernes, Paris, Mignard, 1986, pp. 213-214).

On comprend que l'idéologie spontanée de l'héritier culturel qui vécut en osmose si totale avec l'Ecole le rende aveugle aux conditions symboliques et pratiques d'une pratique.

(10) H. MITTERAND, « Les obsédés de l'objectif, L'enseignement du français en question », Le Débat, 71 , septembre-octobre 1992, pp. 164-172.

(11) L'auteur cite et reprend à son compte les analyses de G. STEINER, Réelles présences. Les arts du sens, Paris, Gallimard, 1991, pp. 187-188.

La médiation du seul discours scolaire légitime, en retombée du "commerce intime avec les œuvres", a été analysée jadis par J. DUBOIS et J. SUMPF dans un article qui conserve son actualité et sa pertinence : "L'acte pédagogique est un discours et, à son tour, le professeur demande à ses élèves de tenir un discours sur l'œuvre, ni plus ni moins » ( Un modéle d'enseignement du français : analyse linguistique des rapports d'agrégation et du capes », Langages, 12, "Linguistique et littérature », 1968, pp. 27-44).

(12) P. BOURDIEU, Les Règles de l'art, p. 418.

(13) D. PENNAC, Comme un roman, Paris, Gallimard, 1992. Les citations qui suivent se trouvent respectivement aux pages $17-18,118-120,132$ et 130-131. Le corpus de romans retenus par Pennac est lui aussi, bien sûr, parfaitement légitime. 
compte c'est moins l'histoire que « l'histoire racontée par quelqu'un ». Le maître est un médiateur qui met en présence, c'est un « marieur » dit même Pennac. Le programme sera d'ailleurs traité car le professeur « entame-t-il un roman à voix haute qu'on se précipite en librairie pour s'offrir la " suite " avant le cours suivant ". Et le romancier d'interpeller les bibliothécaires : "Soyez conteuses - magiciennes - et les bouquins sauteront directement de leurs rayons dans les mains du lecteur ».

Certes les sociologues ont montré l'importance de l'exemple et du relationnel dans la diffusion d'une pratique culturelle en milieu scolaire (14) mais on ne saurait confondre magie des rapports affectifs et formation, incitation généreuse et construction personnelle, animation et apprentissages, intérêt passager et pratique autonome.

\section{Le rapport ordinaire}

Il est enfin un dernier type de discours et de pratique dont la banalité masque ou même vide de son sens la problématique de la médiation (15).

Le premier exemple est celui d'une pratique pédagogique fort commune qui consiste à court-circuiter les démarches d'appropriation matérielle et symbolique du livre. Les manuels de textes sont ainsi octroyés, en attente de lecture sinon de véritables lecteurs, les séries pour l'heure de lecture suivie sont consciencieusement stockées au C.D.I et souvent, "pour simplifier », le professeur prend en charge l'achat groupé du livre trimestriel ( " II connaît bien le libraire, qui fera un prix »).

Une variante de ce scénario consiste à fournir aux élèves, en début d'année, une liste bibliographique dont l'ascétisme informationnel n'a de sens que pour ceux qui sont en connivence culturelle :

\section{Bibliographie-Elèves $6^{\mathrm{e}}-5^{\mathrm{e}}$}

Andersen - Contes

Aymé - Contes du chat perché

Les bottes de sept lieues

Anne Franck - Journal

Garfield - Black Jack

Bradbury - Coup de tonnerre

Joffo - Un sac de billes

Kessel - Le lion

Berna - Le cheval sans tête

Bombard - Naufragé volontaire

Christie - Les dix petits nègres etc.

\section{Defoe - Robinson Crusoe}

R. Fallet - Bulle

Bastia - Le cri du hibou

- Un homme contre la ville

London - Croc blanc

Melville - Moby Dick

H. Malot - Sans famille

(14) Voir par exemple N. ROBINE, Les jeunes travailleurs et la lecture, Paris, La Documentation française, 1984, pp. 176-177 ou F. de SINGLY, Lire à 12 ans, une enquête sur la lecture des adolescents, Paris, Nathan, 1989, pp. 64-65.

(15) Dans sa version journalistique, pour exercer le nouveau métier de médiateur culturel auquel prépare quelques universités, "il faut être passionné par l'art et posséder le goût de la communication », ni plus ni moins (Ouest-France, juillet 1993). 
Ce type de "bibliographie ", mixte d'académisme et d'élitisme, réellement distribuée dans les classes - bien rares sont sans doute ceux d'entre nous qui n'ont jamais rédigé une « information « semblable - se déploie, monotone et terriblement «scolaire", sur deux colonnes et deux pages, sans autre forme de guidage.

Cette offre en « clin d'œil », pseudo-libérale, pléthorique (119 titres cités) et apparemment arbitraire, ne propose aucun des indices de sélection sur lesquels les jeunes lecteurs s'appuient ou pourraient être conduits à s'appuyer (genre, prix, nombres de pages, édition, collection, illustration, thème dominant, date de publication, disponible au CDI, etc.). On imagine certes tout ce que ce genre de document doit à la bonne conscience professorale mais aussi les rancœurs misérabilistes qui peuvent en découler si par malheur le maître s'enquiert quelques mois après de l'usage qui en a été fait, réellement ("On a beau se décarcasser pour eux, ils s'en moquent pas mal ; c'est des perles pour les cochons... j'en ai marre! »). Autrement dit, " offrir de lire sans donner tous les moyens de lire vraiment est non seulement inutile mais néfaste. Une politique culturelle qui veut ignorer cette donnée de base ajoute à son inefficacité les inconvénients de l'incompréhension, puisqu'elle porte tout naturellement à mépriser et parfois culpabiliser les groupes sur lesquels échoue sa généreuse naïveté : puisqu'on leur donne à lire et qu'ils " savent " lire, enrage-t-on, c'est qu'ils ne veulent pas. Résistance perverse ou indignité culturelle, la conclusion s'ensuit vite dans l'esprit de l'offreur voire dans l'esprit des non-lecteurs euxmêmes : prédication inefficace d'un côté, crispation, voire rebellion ou découragement et auto-dévalorisation de l'autre, créent les conditions du malentendu parfait » (16).

Un deuxième exemple de médiation à sens unique (c'est-à-dire qui ne prend pas en compte la réalité culturelle des apprenants) est celle qui sous-tend d'innombrables discours naïvement ethnocentristes ; ils trouvent leur expression dans la théorie misérabiliste du manque et leur complément dans l'optimisme un peu béat de la diffusion.

Soit cette affirmation relevée dans une revue professionnelle : "Le CDI est fondamentalement [...] un lieu de flânerie culturelle. Dans les collèges ruraux, il est le plus souvent le seul lieu de la région où s'exprime une culture littéraire, artistique ou scientifique »(17). Cette perception serait intéressante si elle complétait le constat d'éloignement relatif de la culture légitime par la prise en compte - fût-ce pour la modifier - de la culture au quotidien dans les campagnes (services des B.D.P. et des petites bibliothèques municipales, lecture intensive de la presse régionale et de magazines divers, abonnement à des clubs du livre, consommation importante d'une littérature de masse et de fictions télévisées, intérêts musicaux chez les jeunes, culture pratique, artisanale et technique chez les ruraux en général, etc.).

(16) J.-CI. PASSERON, Le Raisonnement sociologique, Paris, Nathan, Essais et Recherches, 1991 , p. 340.

(17) Inter-CDI, mars-avril 1988, p. 8. Ce socio-centrisme culturel se retrouve constamment, par exemple, dans le pamphlet de M. SCHNEIDER (La Comédie de la culture, Paris, Le Seuil, 1993, "Du culturel », pp. 66-70) qui pose le problème de la démocratisation de la culture mais pas celui de la démocratie culturelle; on reste d'ailleurs pantois devant les variantes adulto-centristes de ce point de vue : « [...] je pense que le jazz est bien, pour l'essentiel, un art, ce que n'est pas, pour l'essentiel, le rock, parce qu'en tant que genre le premier a généralement une valeur esthétique que l'autre n'atteint qu'à l'occasion "...

Pour une approche de " l'esthétique pragmatiste qui rompt avec l'idenfication restrictive de l'art aux seuls beaux-arts et reconsidérer la notion d'art en libérant celui-ci du carcan qui le libère de la vie ", on se reportera à l'étude décapante de Richard SHUSTERMAN, L'art à l'état vif, la pensée pragmatiste et l'esthétique populaire, Ed. de Minuit, coll. Le sens commun, Paris, 1991. 
Ce n'est pas par œcuménisme culturel ou populisme que nous rappelons la différence (dans la domination) c'est tout simplement parce que pour jouer des réticences, des résistances tout comme des engouements superficiels, le médiateur culturel doit apprendre à connaître et à reconnaître les logiques des sub-cultures sous peine, comme dit J.-Cl. Passeron (18), de transformer en refus systématique, violent ou obtus, ce qui n'était peut-être qu'indifférence polie ou laborieux travail d'acculturation.

La fermeture relative mais tendancielle du sous-champ de la littérature de jeunesse tend enfin un dernier piège au médiateur. Max Butlen a signalé dans un article très documenté de la revue Argos « les effets pervers de la légitimation de la littérature pour la jeunesse ». En effet, si les médiateurs de cette littérature ont réussi à construire un territoire et en à faire reconnaître la richesse présente ou potentielle, leur ardent désir de légitimité les expose au risque de "l'enfermement dans des réseaux spécifiques de communications très internes au groupe mobilisé » (19).

II conviendrait d'ajouter que c'est aussi, parfois, un enfermement dans un système de valeurs ou de représentations qui non seulement n'est qu'un système de valeurs parmi d'autres mais encore peut aller jusqu'à travestir la réalité. La survalorisation d'une nouvelle trilogie de valeurs qui conjugue psychohumanisme, ludisme et esthétisme et qui s'oppose au triptyque ancien (moralisme-civisme-didactisme) conduit par exemple à la rédaction de notices descriptives qui occultent des dimensions importantes des textes. Ainsi Villa Aurore de Le Clezio sera présentée sous la plume de médiateurs spécialistes de littérature de jeunesse comme une nouvelle psychologique qui «évoque dans une atmosphère nostalgique et poétique le passage difficile de l'enfance à l'âge adulte », " la transformation des lieux chers à l'enfance des personnages » ou encore comme un récit sur des « lieux que le temps va détruire » alors que c'est inextricablement l'histoire d'une mainmise violente de décideurs, de promoteurs et de bâtisseurs sur la maison d'une dame vieillissante, apeurée et sans défense (20).

Il y aurait beaucoup à dire aussi sur l'image romanesque et stéréotypée de l'écrivain (et de l'écriture) que donnent souvent les magazines littéraires pour la jeunesse : "Poète à quinze ans, mort en duel à trente-six ans, Alexandre Pouchkine vécut sa courte vie comme un roman plein d'aventures et de passions ». Ou encore : Stevenson et un jeune enfant s'amusent à imaginer une

(18) J.-CI. PASSERON, op. cit., chapitre XIV, « Le polymorphisme culturel de la lecture », pp. 335-345.

(19) M. BUTLEN, “Chroniques d'une légitimation ", Argos, № 5, novembre 1990, pp. 52-64 (sur "l'effet réseau " et sur "l'effet bouclage ", voir les analyses d'A. CAUQUELIN, L'art contemporain, P.U.F. coll. Que sais-je, № 2671, Paris, 1993, chapitrell).

Un libraire qui se définit lui-même comme un "médiateur personnalisé " donne un étonnant exemple de cette prétention à l'auto-légitimation et au monopole de la légitimité culturelle ; il regrette en effet que " très souvent, malheureusement, les parents fassent obstacle (au choix des jeunes) en jugeant de l'utilité ou de l'inutilité de tel achat, en termes de valeurs de référence par rapport à l'école ou à la culture [...]; en fait, par son désir de faire partager son plaisir de lire, le libraire va amener le client à acheter tel livre plutôt que tel autre " (“Profession : libraire », Le Français aujourd'hui, «Lieux de lecture », juin 1993, № 102, p. 85).

II serait intéressant de repérer systématiquement et d'analyser les enjeux et les effets de ces très nombreuses tentatives de monopolisation de prescription culturelle ou de substitution de responsabilité éducative : «Popi a une influence positive sur ma petite fille Myriam. Après la lecture d'un numéro où l'on voit Léo descendre de son lit, elle a demandé à quitter son lit à barreaux pour dormir dans un lit d'où l'on descend " - Nathalie, maman de 3 enfants. "J'aime tout dans Youpi. Maintenant, je sais comment se fabrique le fromage et j'aimerais savoir comment se fabrique le papier. Ma maman ne m'explique pas toujours si bien " - Nicolas, 5 ans 1/2 (extrait du courrier publicitaire de Bayard Presse Jeune, 23 août 1993).

(20) J.M.G. Le CLEZIO, Villa Aurore, Paris, Galimard, coll. folio junior, № 2, 1985 "Livres en échos, sélection de livres pour adolescents ", C.R.L.I.J. Rhône, 1986, p. et " 542 romans, contes et albums pour 13-16 ans", CRLIJ-Loire, BM de Saint-Etienne, 1989, p. 5. 
île dont le petit «Llyod dessine les contours sur une feuille de papier. L'écrivain note "L'île au trésor » sur la carte. Soudain les personnages du roman semblent surgir devant ses yeux. Quelques instants plus tard, il a déjà commencé à écrire I'histoire. Elle sera terminée en quelques mois. Le succès... » (21).

Rapport im-médiat, rapport fétichisé, rapport privilégié ou rapports ordinaires ont en commun une surfocalisation sur l'objet littéraire légitime et une attention trop distraite et trop discrète à la diversité des jeunes lecteurs et aux conditions culturelles de développement d'une pratique "significative ", au double sens du terme.

C'est précisément pour rompre avec ces conceptions que nous faisons appel aux disciplines socio-historiques et ethnologiques.

\section{QUELQUES COMPOSANTES CONSTITUTIVES D'UNE PRATIQUE CULTURELLE}

« La très ordinaire histoire d'Alain et d'Ali » nous servira à la fois d'illustration et de matériel d'analyse.

Convenons qu'Alain et Ali sont deux élèves de $5^{e}$, dans un collège des Minguettes où je travaillai naguère ; ils m'accompagnent jusqu'au portail, un mercredi à midi, à la fin des cours. Disons qu' Alain est spécialiste des corners et des tirs au but et qu'Ali, lui, roi des dribbles, est le meneur de jeu de son équipe. Voici en substance ce qu'ils me racontent (ils sont loquaces, comme on va voir).

Chaque mercredi et le samedi après-midi ou le dimanche matin durant toute la saison, en fait dès qu'ils ont un moment, ils jouent au foot (sans compter les petites parties improvisées à l'école, pendant les récréations).

Ils font partie d'une équipe, s'entraînent sur le terrain voisin et dans les moments de liberté s'amusent avec les copains, " taquinent " la balle au pied de l'immeuble.

Quand les pères ont un peu d'argent ou qu'ils ont une invitation du Service Sport et Culture de la ville, ils assistent à Gerland à un match, dans les tribunes populaires, dans les virages, toujours au même endroit si possible.

Quand l'équipe de leur club joue à domicile, ils vont en bande ou avec leurs grands frères, voir le match et encourager leur équipe.

Ils ajoutent qu'ils ont monté une petite loterie de quartier avec un travailleur social pour s'acheter une tenue, des maillots, des chaussures à crampons et même des genouillères pour le gardien.

Ali, lui, a demandé à sa mère de lui confectionner le brassard de capitaine. Sa mère râle d'ailleurs chaque lundi quand il faut lui laver maillot et chaussettes et elle s'inquiète lors de chaque match car elle craint l'accident pour le gamin qui « risquerait en plus de manquer l'école "...

Ils ont des réunions informelles à la sortie de l'école pour savoir qui va jouer et à quel poste lors du prochain match et dans les rangs, en attendant de " monter en cours », il leur arrive de discuter des résultats des équipes professionnelles aux différents championnats et coupes.

(21) Je bouquine, № 105, novembre 1992, p. 93 et Je bouquine, № 114, août 1993, pp. 85-88. 
Ils savent d'ailleurs très bien en général où on en est du classement de la D1 (première division), si Monaco s'est qualifié et que c'est Platini qui a été le dernier porteur de la flamme olympique...

Ils parlent arbitre, règlements, tirage au sort, petit pont, tir en lobe, tactique du 4-2-4, carton jaune, mi-temps, temps règlementaire, remplaçant, etc, etc. Ils savent aussi que le mot « football » est d'origine anglaise, que Le Havre fut le premier club français professionnel, que le maillot de Marseille est blanc et bleu et que " c'est vachement chouette, au Stade Vélodrome, la pelouse sous les projecteurs, quand ils jouent en nocturne »; ils n'ignorent pas que Tapie a vendu à l'Inter de Milan (ou au Milan A.C., ils ne sont pas d'accord sur ce point) Jean-Pierre Papin (lequel JPP, l'autre jour, a marqué un super but en ciseau retourné), etc.

Ils me disent que leurs pères jouaient aussi au ballon et qu'ils aiment bien çà parce qu'on rigole et qu'on se défonce et qu'ils se laissent pas faire quand il y en a un qui joue dur, même si en fait c'est eux qu'on craint parce qu'ils sont des Minguettes...

Alain demande à sa mère de lui acheter des «Vache Qui Rit » parce qu'il y a souvent des images de footballeurs qu'il collectionne et échange avec Ali ; quand il va chez le dentiste ou chez le médecin et qu'il y a un «France Football » qui traîne, il aime bien le regarder ; d'ailleurs il a repéré “ L'Année du foot » au CDI et quand il a permanence il va le feuilleter avec son copain. A son anniversaire ou à Noël, il a demandé un ballon, un jeu video sur le foot ou un bouquin sur les «Grandes Heures du football ».

Quand il va chez le coiffeur tout seul, il ne dit rien, mais « il aime bien quand les grands parlent foot » II préfère Thierry Roland à Jean-Michel Larqué (c'est l'inverse pour Ali) qui commente drôlement bien les matches sur la Une et de toute façon il ne loupe pas «Télé-But » et le « Journal du foot " sur la 2.

L'an passé, ils ont gagné un tournoi de sixte et ils ont chacun un fanion et une petite coupe dans leur chambre, près d'eux.

Bien sûr, ils ne seront pas des professionnels mais ils disent qu'ils gagnent beaucoup d'argent, ont de belles voitures, font des voyages et tout...

Etc, etc, etc...

Autrement dit, (si l'on passe de la reconstitution ou du reportage ethnographique à l'analyse proprement ethnologique), cette pratique footballistique génère et est générée par un capital culturel fait de personnes multiples et de sociabilités diverses, d'objets fonctionnels ou symboliques et de gestes codés ou inventés, d'images et d'imaginaires, de lieux et de temps, de discours et de rites, de savoirs et de représentations, de valeurs et d'enjeux.

A l'évidence, nos petits footballeurs sont, dans leur domaine, des spécialistes sinon des érudits (on pourrait dire la même chose pour les filles si on prenait l'exemple des chanteurs ou des élèves de lycée professionnels pour les motos).

On n'imagine pas non plus qu'ils soient de bons petits footballeurs (au sens technique du mot) sans que leur pratique ne soit en même temps constituée et construite et par des personnes et des rencontres, et par des manières de dire et par des manières de faire, et par des habitudes et par des savoirs, etc.

Autrement dit encore, leur culture footballistique est à la fois très structurée (ce n'est pas n'importe quoi) et structurante (“aller au foot » n'est pas « aller à la bibli » : ni les valeurs, ni les discours, ni les imaginaires, ni les sociabilités, ni 
les temps, ni les connaissances, etc., ne sont les mêmes, même si elles ne sont pas nécessairement exclusives).

C'est un capital culturel avec son état institutionnalisé (le championnat, les coupes, les titres, les classements, les catégories, etc.), son état objectivé (les ballons, les maillots, les revues, les terrains, etc.) et son état incorporé (« tout jeune j'avais déjà un ballon à la place de la tête " confie Platini dans son autobiographie), bref avec l'habitus qui convient.

On a donc compris que le développement d'une pratique de lecture engage l'ensemble organisé de ces paramètres qui procède non seulement d'une "curiosité construite » (22) mais véritablement de la mise en place d'un habitus lectural (23).

\section{PROPOSITIONS ETHNO-DIDACTIQUES}

Or, il semble bien que quand il est question aujourd'hui du lecteur et du lecteur en formation, d'un habitus de lecture, c'est-à-dire d'un ensemble acquis et structuré d'aptitudes, d'attitudes et d'habitudes qui interagissent systématiquement, les pratiques scolaires d'apprentissage présentent souvent cinq caractéristiques peu satisfaisantes (du moins au regard de l'analyse ethnologique des conditions de développement d'une pratique culturelle). Ces cinq paramètres "se tiennent ", constituent un système didactique qui touche aux temps, aux objets, aux modalités, aux problématiques culturelles et enfin aux contextes d'apprentissage.

Dans une problématique " socio-didactique " ou même " ethno-didactique ", les pratiques scolaires archi-dominantes sont en effet trop ponctuelles, trop partielles, trop magistrales, trop légitimistes, trop décontextualisées enfin. Nous procéderons à un rapide examen critique de ces traits et proposerons des éléments de réflexion et d'action pour mettre en place un autre système didactique, certes beaucoup plus exigeant mais qui devrait permettre aux équipes d'enseignants et à leurs partenaires du livre d'analyser leurs pratiques, et aux comportements culturels du plus grand nombre de mieux se développer.

$1^{\circ}-$ Le travail d'institution des lecteurs est trop ponctuel. Le plus souvent on se satisfait d'un peu d'initiation culturelle ou de familiarisation avec la culture du livre, ses codes et ses langages en début d'année ou en fin de manuel, ou encore à l'occasion d'un projet inédit, travail épisodique et en miettes, en tout cas sans suivi ni reprises dans le cursus.

Cette bonne volonté didactique repose sur l'idée qu'un savoir élémentaire et restreint suffit, qu'il trouve d'emblée sa formulation et son champ d'application

(22) M. POULAIN, «Lecteurs et lectures: le paysage général ", Pour une sociologie de la lecture, Lectures et lecteurs dans la France contemporaine, Paris, Editions du Cercle de la Librairie, coll. Bibliothèques, 1988, pp. 40-41.

(23) J.-M. PRIVAT et M.-C. VINSON, « Habitat vertical et habitus lectural », Pratiques, № 52, 1986.

Dans la même perspective, N. ROBINE conclut son étude « socio-psycho-ethnograhique " en écrivant que " toute opération de lecture apparaît comme un acte anthropologique. Toute lecture, dans tous les sens que le terme peut prendre, se situe dans un moment de l'histoire sociale, culturelle et psychologique du lecteur. Ce moment ne peut être compris que s'il est replacé dans la sphère des relations que le sujet entretient avec les institutions et les individus qui gravitent autour de lui. Dans la relation à la lecture, se retrouve la totalité de l'histoire personnelle du sujet. Inversement, établir la relation du sujet à la lecture, c'est faire l'histoire des groupes sociaux dans lesquels il a évolué " (Les jeunes travailleurs, p. 199). 
définitifs, que ce savoir ou ce savoir-faire se structurent aisément et une bonne fois pour toutes.

"L'initiation au C.D.I en $6^{\mathrm{e}}$ ” pourrait être l'exemple type de ce paradoxe pédagogique pour qui introduire seulement aux premiers éléments d'une pratique (ce qu'est par définition une initiation) suffit (il y a rarement en $5^{\mathrm{e}}$ et au-delà une suite à cette « initiation ") ; les sociologues de la culture ont montré pourtant qu'entrer dans la société des lecteurs exige un rituel beaucoup moins expéditif et les historiens attestent tous que les comportements culturels se modifient lentement, que les évolutions des groupes comme celles des individus procédent par étapes et que ces changements n'ont lieu «qu'au sein d'un ensemble de processus dont les différentes régulations sont interdépendantes et successives $\gg(24)$.

II faudrait donc, au contraire, mettre en place un processus continu, une dynamique structurelle, c'est-à-dire inscrite et construite sur la durée (pour durer) et dans des situations diversifiées, considérant par là même que tout véritable apprentissage est le fruit d'un processus progressif d'élaboration et d'ajustement et que l'apprentissage culturel est constitutif du développement des compétences du lecteur en général.

Les éditeurs qui publient "Popi, le journal de tous les bébés », et les bibliothécaires qui parlent de bébés-lecteurs et organisent des colloques sur le sujet le savent bien (25), tout comme les enseignants de maternelle qui travaillent avec les lecteurs en herbe dans les marmothéques de l'école et comme les médiateurs du livre qui fréquentent les salons du livres pour tout-petits. Mais pourquoi donc commencer si tôt... et le plus souvent s'arrêter si vite, à l'Ecole du moins (26)!

Se limiter à une trop rapide propédeutique culturelle, au lieu d'aider tous les enfants "à se construire leurs conduites de lecteurs de livres " (27), pourrait avoir l'effet socialement pervers de " bénéficier sélectivement aux enfants de milieux favorisés, au lieu d'aider prioritairement les enfants moins familiarisés avec le livre et l'écrit » (28).

En effet, le processus de socialisation culturelle, inauguré par l'acte de marquage instituant un individu lecteur (par exemple), « avec tous les privilèges et toutes les obligations corrélatives ", produit des effets bien réels à condition d'être "durablement inscrits dans le corps et dans la croyance ". "L'institution n'est complète et complètement viable que si elle s'objective durablement dans la logique d'un champ " (si elle est d'abord prolongée, renforcée, confirmée par le champ didactique) et si les agents sont en mesure " d'habiter ", comme dit Bourdieu, les institutions de lecture, c'est-à-dire d'en incorporer les exigences

(24) N. ROBINE, "L'évolution de la lecture des jeunes d'après les enquêtes françaises ", Pratiques, № 61, mars 1989 , p. 125.

(25) Voir par exemple Les Livres à petits pas contés, Actes du colloque de Villeurbanne, mai 1992, Villeurbanne, Maison du Livre de l'Image et du Son, 1993

(26) Une enquête par questionnaire que nous avons menée dans cinq académies pour le compte de la F.F.C.B. (Fédération française de coopération entre bibliothèques) sur le thème des relations entre monde scolaire et bibliothèques publiques montre clairement que de la petite section de maternelle aux classes terminales des lycées la coopération entre Ecole et Bibliothèques diminue trés réguliérement, jusqu'à une quasi absence de liens, avec des pointes d'intensité trés nettes en $6^{\mathrm{e}}$ et en $2^{\mathrm{e}}$.

(27) F. SUBLET et Y. PRETEUR, "Les conduites réflexives d'enfants de 5 à 6 ans vis-à-vis des livres de jeunesse ", Revue Française de Pédagogie, № 85, octobre-novembre-décembre 1988, pp. 47-54.

(28) Y. PRETEUR et L. ROUQUETTE, "L'incidence d'une pédagogie fonctionnelle autour du livre de jeunesse sur les compétences orales et écrites d'enfants de grande section maternelle de milieux sociaux contrastés ", Revue Française de Pédagogie, № 98, janvier-février-mars 1992, pp. 29-40. 
selon la logique d'une histoire personnelle et particulière, loin des conformisations superficielles ou sporadiques de la conduite (29).

Or, l'Ecole, contrairement aux bibliothèques publiques par exemple, a le « temps » pour elle; elle peut donc poser le problème du rythme et de l'ampleur de l'acculturation. Ce travail de dé-construction partielle et éventuelle d'un habitus primaire (celui d'Alain et d'Ali probablement) et de reconstruction d'un habitus secondaire (davantage tourné vers le livre (30)) prend du temps dans la mesure où, "par le " choix " systématique qu'il opère entre les lieux, les événements, les personnes susceptibles d'être fréquentées ", I'habitus engage l'intégrité et l'intégralité de la personne qui " tend à se mettre à l'abri des crises et des mises en question critiques [...] » et à s'assurer par des stratégies pensées ou impensées d'évitement (que les pédagogues connaissent bien) un " univers relativement constant de situations propres à renforcer ses dispositions en offrant le marché le plus favorable à ses produits 》(31).

Au risque de trop raisonner par analogie, j'illustrerai volontiers mon propos par l'histoire mythique de La Nef ARGO : c'est l'histoire du vaisseau Argo (lumineux et blanc) dont les Argonautes remplaçaient peu à peu, au cours de leurs libres pérégrinations et de leurs différentes routes imposées, chaque pièce, au gré des tempêtes, des usures et des bricolages plus ou moins inspirés et forcés.

En sorte qu'ils eurent pour finir un vaisseau entièrement nouveau, sans avoir à en changer ni le nom ni l'allure générale.

Ce vaisseau Argo est bien utile : il fournit l'allégorie d'un sujet éminemment culturel dont l'acculturation serait à la fois totale, continue et heureuse : à force de substitutions et de combinaisons de traits culturels nouveaux avec des plus anciens, Argo conserve son identité tout étant un autre...

Il convient donc, particulièrement pour les apprentissages culturels, d'abandonner les pédagogies qui reposent sur des conceptions behavioristes (du simple et de l'analytique au complexe et au synthétique) ou maturationniste (du b.a. ba de la technique, au cp, au supplément d'âme culturel, au lycée, de la lecture aux lectures donc) et de mettre en œuvre des démarches socio-constructivistes qui prennent en compte la co-construction des compétences technicoculturelles.

D'ailleurs, les nouvelles directives, tant pour l'enseignement élémentaire et primaire que pour le secondaire encouragent (encouragent seulement) à suivre cette voie : " apprendre à vivre au milieu des livres et des autres écrits ", " découvrir les espaces privilégiés du livre et les gestes "cultivés" de la lecture ", " participer à des échanges, des discussions sur les livres ", " capitaliser de nombreux savoirs implicites sur les façons de vivre au milieu des

(29) Les bibliothèques publiques ont à mener un difficile mais nécessaire « travail de fidélisation ": des études récentes ont montré la place croissante des bibliothèques dans le maintien ou le renforcement des pratiques de lecture (F. de SINGLY, Les jeunes et la lecture, chapitre VI, "Les vertus des bibliothèques », et notamment le point VI-4 "Assurer un suivi des usagers ", pp. 176-182, Ministère de l'Education nationale et de la Culture, Les dossiers Education et Formations, 24, janvier 1993).

(30) De toute façon, que la prime socialisation culturelle (famille et groupe de référence) soit ou non retravaillée, le travail d'assimilation culturelle " coûte du temps et du temps qui doit être investi personnellement par l'investisseur » (P. BOURDIEU, "Les trois états du capital culturel ". Actes de la recherche en sciences sociales, 1979, №30, pp. 3-6) contrairement aux théories psychologiques qui voudraient que " tout se joue dans la prime enfance". Autrement dit, le travail que nous proposons s'adresse à tous, même si sa mise en $œ u v r e$ et ses effets doivent être socialement différenciés.

(31) P. BOURDIEU, Le Sens pratique, Paris, Minuit, 1980, p. 102. 
informations et des réalités culturelles qui nous entourent " sont quelques-unes des compétences que les trois cycles de l'école visent à développer de façon régulière et continue (32) ; au collège, " tout professeur, quelle que soit sa discipline " est invité à s'intéresser "à la diversité des livres et des institutions qui les produisent ", à favoriser « la constitution d'une bibliothèque personnelle, l'exploitation du CDI, les ressources des bibliothèques de la classe, du collège, du quartier, de la ville et des librairies » (33).

L'existence d'une Mission lecture pour les étudiants repose enfin sur cette juste idée que « la familiarisation avec l'écrit est un processus continu » et qu'il est illusoire de penser que si les étudiants « ne recourent que très peu au conseil des bibliothécaires et des libraires, c'est parce qu'ils maîtrisent parfaitement les catalogues, les classements et les espaces » (34).

En résumé, contrairement à ce que laisserait croire un certain bon sens doublé d'une illusion techniciste, il faut commencer tout de suite les apprentissages liés à la culture du livre et à la culture du lecteur (bien avant que l'enfant ne maîtrise le code de l'écriture) et contrairement à l'illusion instrumentaliste, il faut, selon nous, abandonner l'idée que quelques informations rapides et élémentaires suffisent à la mise en place définitive d'un comportement de lecteur, le prestige du livre et de la lecture faisant le reste.

\section{2o- Le travail d'institution des lecteurs est trop partiel.}

On n'étudie guère que la couverture avec parfois une excursion du côté des index ou des bibliographies ; on se centre donc, au mieux, sur l'objet-livre en soi mais on ne s'intéresse qu'exceptionnellement aux lieux d'offre et à leurs codes d'accés, aux intermédiaires de lecture et à leur vocabulaire, aux protocoles sociaux du rapport aux livres et à leurs conventions, aux trajectoires personnelles des lecteurs et à leur logique, etc.

II suffira ici de rappeler la thèse "systémique » que M. Poulain avance avec force dans l'introduction à l'ouvrage collectif Pour une sociologie de la lecture:

«La lecture n'est pas seulement le moment où celle-ci s'effectue, mais un ensemble, "un corps de pratiques": tout ce qui la conditionne, y prépare, y conduit, la prolonge ou l'annule n'est pas périphérique à la lecture mais en est radicalement constitutif [...]. Tout en effet, circonscrit une lecture avant même qu'elle ne s'effectue. La lecture n'est que l'acte ultime d'une série de manipulations apparemment spontanées et conjoncturelles qui mettent un livre entre les mains de son lecteur. Comment ce livre est-il choisi ? D'où vient-il ? De qui vient-il ? A-t-il été acheté ou était-il dans la bibliothèque familiale ? Est-il passé entre plusieurs mains ? Fait-il l'objet de conversations, de différentes formes de sociabilité ou est-il entièrement intériorisation et activité solitaire?

(32) Ministère de l'Education nationale et de la Culture, Direction des écoles, La Maîtrise de la langue à l'école, C.N.D.P.-Savoir Livre, collection Une école pour l'enfant, Des outils pour les maîtres, Paris, 1992, passim.

(33) Ministère de l'Education nationale, Direction des Collèges, Compléments aux Programmes et Instructions, Lire au Collège, s.d., p. 2.

(34) E. FRAISSE, « L'université au miroir de la lecture », Esprit, № 194, août-septembre 1993, pp. 139 -141 (voir aussi, sous la direction d'E. FRAISSE, Les étudiants et la lecture, P.U.F., Politique d'aujourd'hui, Paris, 1993). C'est depuis une bonne quinzaine d'années que les anglo-saxons réfléchissent sur (et travaillent à) l'éducation de l'usager des bibliothèques et notamment des étudiants ; citons en guise de bibliographie sommaire, John LUBANS, Jr., ed. Progress in Educating the Library User, R.R., Bowker Company, New-York, 1978 ou N. FJALLBRANT and I. MALLEY, User Education in Libraries, second edition, Clive Bingley, London, 1984. Sur la formation des usagers (buts et objectifs) comme partie intégrante du cursus universitaire (et du fonctionnement optimal de la bibliothèque), voir le programme très précis des apprentissages à court et long termes que propose A. DAUMAS, Bulletin des Bibliothèques de France, t. ,6 № 1984, pp. 562-567. 
Et comment choisir un livre lorsqu'on est confronté à une masse devant laquelle on ne dispose d'aucun repère, lorsqu'un titre égale un autre titre, lorsque rien n'introduit de différence, donc de préférence ? Le lecteur assidu, le familier de la lecture ignore même tout ce qu'il met en jeu dans le choix d'un livre : connaissance de l'auteur, de l'éditeur, de la collection, lecture en diagonale de la quatrième de couverture, évocation de lectures antérieures sur un sujet proche, consultation éventuelles de critiques, conversations avec des proches. Ce qu'il ressent comme une curiosité individuelle est une curiosité construite [...]. Le familier de la lecture met en jeu dans ses choix - puis dans ses lectures - un constant système de questionnements et de réponses, d'allers et de retours entre les offres de lecture et lui-même, une grande entreprise de références où tout livre en évoque toujours un autre, toute lecture une autre » (35).

Autrement dit, la conduite lecturale est toujours médiate (36), même et surtout dans les cas où la pratique, au terme d'un long, lent, diffus et complexe processus d'incorporation et d'intériorisation est vécue comme « naturelle ». Dans cette perspective, on voit que l'intensité de la pratique culturelle a partie liée à la fois à la durée des sollicitations et des apprentissages mais aussi aux cumuls des expériences et des situations. En effet, à la différence des besoins primaires (boire, dormir), la pratique culturelle se nourrit d'elle-même, autrement dit "s'accroît à mesure qu'elle s'assouvit, puisque chaque nouvelle appropriation tend à renforcer la maîtrise des instruments d'appropriation et, par là, les satisfactions attachées à une nouvelle appropriation » (37).

Malheureusement une loi de la didactique culturelle veut que l'absence de pratique s'accompagne généralement (chez l'enseigné comme chez l'enseignant...) de l'absence du sentiment de cette absence, que « la conscience de la privation décroît à mesure que croît la privation "... (38)

On voit donc que si l'on ne s'abandonne pas au défaitisme didactique, le travail doit être conscient et prolongé parce qu'il doit être systématique, c'està-dire, pour reprendre à titre d'exemple les propositions programmatiques d'un autre chercheur, "réaliser avec efficacité un triple repérage : repérage dans les lieux de lecture, repérage dans les objets à lire, repérage dans la pratique personnelle du sujet lisant.

Repérage dans les lieux de lecture : dans la bibliothèque personnelle, en $\mathrm{BCD}$, au CDI, à la bibliothèque ou médiathèque municipale, chez le libraire... ». On sait en effet que « tout ce que les classes favorisées valorisent dans une bibliothèque ou une librairie : l'éclectisme, la variété des choix dans un même genre, le mode de classement des ouvrages ", représente pour les petits lecteurs " des facteurs d'éloignement de ces institutions dont l'agencement est conçu par des lettrés, pour des lettrés » (39).

Cette familiarisation avec tous les espaces de la lecture et ses médiateurs, cette solide connaissance des territoires de la lecture, de ses agents et de leurs langages suppose en second lieu " la liberté d'errer dans le texte, la maîtrise de son organisation en chapitres, l'aptitude à faire des impasses, le pouvoir

(35) M. POULAIN, op.cit., p. 41

(36) Y. REUTER développe le même type d'analyses à propos de la pratique de la littérature qui se vit en toute légitimité comme "négation des médiations " mais qui en fait procéde par " exacerbation des médiations" ("Littérature et médiations culturelles », J.-M. PRIVAT et Y. REUTER (ed), Lectures et médiations culturelles, actes du colloque de Villeurbanne, P.U.L., Lyon, 1991, pp. 59-73.

(37) P. BOURDIEU, L'Amour de l'art, p. 157.

(38) id., ibid.

(39) N. ROBINE, Les jeunes travailleurs, p. 125. 
d'utiliser à bon escient table des matières, sommaire, index, glossaire, préface, notes... toute la périphérie du texte $"$.

La prise de pouvoir sur les lieux, les objets ne saurait se concevoir enfin sans une " pratique réflexive qui conduit chacun à évaluer régulièrement ses stratégies, ses comportements, ses compétences pour bâtir de nouveaux projets de formation et se risquer à découvrir de nouveaux parcours, de nouvelles trajectoires de lecture » (40). Un lecteur se définit en effet non seulement par ses habitudes de lecture (type et rythme de lecture par exemple) mais aussi par son itinéraire de lecture.

3- Le travail d'institution des lecteurs est trop fréquemment transmissif ou simplement incitatif. On pense à tous ces courts-circuits culturels qui, " pour simplifier ", évitent aux élèves de se donner la peine d'aller euxmêmes acheter les livres qu'ils étudient ou à ces visites éphémères d'écrivains dans les classes. On songe aussi à ses innombrables petits ouvrages de vulgarisation qui prétendent suivre l'histoire technique du livre (de la fabrication du papier au bon-à-tirer) dans une forclusion totale des lectorats et ne se doublent donc jamais d'une histoire ou d'une sociologie des pratiques dans laquelle le lecteur pourrait précisément se sentir impliqué.

Les rares manuels scolaires qui prennent en compte quelques caractéristiques de la pratique lecturale procédent en général par « leçons » et suggèrent une approche pauvrement pragmatique de la culture du livre : "Cette couverture vous donne-t-elle envie de lire le roman ? Pourquoi ? En quoi peut-on dire que la couverture est la vitrine du roman? » ou "L'une des éditions guide-t-elle plus son lecteur? Laquelle achèteriez-vous ?"

Il ne sert à rien non plus d'imposer à tout prix, lors de véritables cours de bibliothéconomie, l'apprentissage forcée des codes professionnels d'accés, dans la mesure où l'usage de la logique classificatoire est " une opération hautement qualifiée ». Le forçage est à redouter car si "l'appropriation ne s'installe pas, il y a rejet, désaffection du lieu de lecture. Le lecteur qui ne trouve pas les livres souhaités ne proteste que s'il se confère le droit à la parole et une certaine autorité culturelle ; les autres partent sans dire mot» (41). Le discours proféré par les institutions culturelles est donc double : «créées pour tous, leur système de fonctionnement ne permet qu'à quelques-uns de les fréquenter et parmi ces derniers, un nombre encore plus restreint se moule dans leurs codes et leurs structures ". Autrement dit, dans la mise à la portée des espaces du livre, les enseignants ont un rôle important à jouer « en diffusant le vocabulaire précis du livre et de la lecture, en familiarisant avec les lieux du livre, en apprenant à se servir du livre » afin que progressivement et personnellement s'élaborent "les cadres mentaux et culturels permettant la perception et la maîtrise d'un ordre dans l'accès conceptuel au savoir » (42).

(40) M. BUTLEN, “Les BCD, de l'innovation à la généralisation ", La culture de l'écrit et les réseaux de formation, actes de l'université d'été de Lacanau, sous la direction de M. BUTLEN et J. HÉBRARD, CRDP, Académie de Créteil, coll. Argos, 1992, pp. 261-262.

Pour des pistes de travail plus précises (et facilement transposables aux divers niveaux d'apprentissage), voir M. COUET, «Vers une typologie des activités en BCD ", voir Argos, № 1, mai 1989, pp. 28-33 ou P. CHASSAGNE, J.-P. DEBANC, C. GARCIA-DEBANC, 50 activités autour du livre, dans la classe ou en BCD, Toulouse, CRDP, 1993 (à paraître).

(41) N. ROBINE, "Relais et barrières : la perception et l'aménagement de l'espace et des classifications par les usagers dans les lieux de prêt et de vente du livre ", J.-M. Privat et Y. Reuter (ed), op. cit., pp. 115-126.

(42) id., ibid. 
Les « animations culturelles » (souvent dans le cadre de projets éducatifs) prennent acte du décalage entre un public scolaire désormais élargi et les modes plus ou moins institutionnalisés et inopérants ou insuffisants de transmission ; un effort de modernisation de la communication culturelle est ainsi conduit, souvent sur les modéles utilisés dans les bibliothèques publiques (parfois en coopération avec elles), pour développer la propension à s'approcher et à se rapprocher réellement des biens et des services culturels proposés. Mais cette politique incitative s'apparente souvent, semble-t-il, à une "consommation épisodique de loisirs culturels» (43). Elle se développe à partir d'une prise en compte des modifications de la demande " et contre la manière scolaire de diffuser et donc d'acquérir la culture, mais ne remet nullement en cause »la légitimité de la hiérarchie culturelle «. Par l'animation on entend faire lire « autrement »: " on cherche à jouer sur les attitudes, à inculquer en douceur une disposition cultivée, selon de nouvelles valeurs qui correspondent à l'ethos des nouvelles classes moyennes : l'échange, le plaisir, la créativité » (44). II s'agit moins d'imposer comme dans le modèle magistral et directif que de proposer, de séduire, d'inciter, mais selon le principe du « divertissement guidé » ou de «l'appropriation en vrac » qui implique chez le public soit " un rapport aisé et de connivence avec la culture ", sélectif, soit "un rapport auto-didaxique », rare (45). Autrement dit, pour que ce mode de transmission diffuse ne se résume pas pour le plus grand nombre en une transmission confuse, il conviendrait de " dépasser l'organisation ponctuelle d'activités, assimilables trop souvent à des " coups culturels ", pour les intégrer dans une stratégie générale d'inculcation explicite et systématique des différents codes qui permettent l'appropriation réelle de l'offre $[\ldots] »(46)$.

Un apprentissage réel, surtout en matière culturelle, doit être de type appropriatif (agentif comme l'on dit significativement dans le jargon des didacticiens, acculturatif comme dirait l'ethnologue) c'est-à-dire qu'il doit prendre en compte les représentations et les pratiques des lecteurs, laissant ainsi la possibilité aux stratégies de conversion ou de reconversion de se développer.

II faudrait aussi travailler régulièrement les réinvestissements des apprentissages car on ne " fait » pas les catalogues de librairies comme on " fait »le conditionnel ou l'accord des adjectifs de couleur, à moins de se contenter, là comme ailleurs, de connaissances purement déclaratives. Une " anecdote » pédagogique servira d'illustration vivante à cette idée qui veut que des apprentissages appropriés (au double sens du terme) ont à la fois, et toujours, un caractère linguistique et un caractère culturel, sont multi-dimensionnels; les enfants apprennent en parallèle " et à élaborer des significations à partir du code écrit, et à traiter des masses verbales beaucoup plus longues, inscrites dans des supports et contextes variés, dans des situations diverses de productiondiffusion et réception, relevant de types discursifs et textuels différents et à élaborer des attitudes et conduites culturelles de lecteur dans divers actes de lecture : repérage, choix, achat ou emprunt ; détermination des lieux, moments

(43) B. SEIBEL, Bibliothèques municipales et animation, Paris, Dalloz, 1983, p. 46.

(44) id., ibid., pp. 82-83.

(45) id., ibid., pp. 316-317.

(46) id., ibid., p. 320. 
et modalités de la lecture ; pratique de cette activité et de celles qui l'accompagnent ou la prolongent $»(47)$.

Une demi-classe de $6^{\mathrm{e}}$ participait à un petit jeu proposé par des camarades de $5^{\mathrm{e}}$. Chacun devait, à tour de rôle, répondre à une question de "compétences culturelles » concernant un des ouvrages présentés sur une table d'exposition. Vint le tour d'un jeune élève qui tira la question : "Quelle est l'illustration que l'on retrouve en tête des douze chapitres des Aventures du capitaine Cook? ". Non seulement l'élève manifesta une gaucherie certaine dans l'identification de l'ouvrage mais surtout, le livre enfin repéré, il ne sut qu'en faire. A vrai dire, il ignorait bel et bien ce qu'était un « chapitre »...

La première hypothèse est celle de l'analphabétisme culturel : on ne lui a jamais véritablement appris ce qu'était un chapitre et il est la victime d'une invraisemblable lacune pédagogique, d'un déficit didactique.

La seconde hypothèse renvoie plutôt à la logique de l'altérité culturelle : il y a eu à la fois apprentissage programmé par l'institution et résistance larvée ou têtue de la part de l'élève ; cet apprentissage a comme " glissé » sur un sujet qui se désigne ou se vit comme étranger à la culture écrite scolaire et la refuse, indifférent ou affichant un désintérêt provisoire ou définitif.

La dernière hypothèse, la plus vraisemblable, renvoie à la logique de l'illettrisme culturel : il y aurait eu une certaine forme d'apprentissage puis désapprentissage ; l'élève aurait désappris à la fois parce qu'il y aurait eu dysfonctionnement didactique (on lui a appris trop sommairement, sans réinvestissement fonctionnel en lecture ou en écriture) et absence de relais culturel personnel (apathie du contexte culturel, absence de situations de réemploi).

On imagine ce que peut être l'écoute et la compréhension de cet enfant lorsque son professeur d'histoire, de mathématiques ou de sciences lui demande de prendre son livre au chapitre 7,8 ou 11 ou lorsque le documentaliste lui suggère le choix de tel ouvrage parce qu'il n'a que six courts chapitres...

Il doit en aller de même lorsqu'il est question de biographie ou de bibliographie, d'index ou de glossaire, de première ou de quatrième de couverture, de collection ou de genre, etc.

L'appropriation serait certainement favorisée par des pratiques qui donnent toute son importance à l'écriture dans la structuration des apprentissages culturels et méta-culturels (ou métalecturaux). Tenir son journal de lecteur est une de ces activités "synthétiques » ou «panoramiques » qui peut permettre au lecteur de se construire une identité... de lecteur ; on peut aussi apprendre à affiner (diversifier et optimiser sa perception de l'offre par exemple) ses stratégies personnelles d'appropriation d'un espace culturel (librairie ou bibliothèque), ses stratégies de lecteur en situation donc, exactement comme on apprend à l'élève à accommoder méta-cognitivement ses stratégies de lectures en fonction de projets ou d'objets de lecture différents.

Dans chaque cas, il s'agit de prendre appui sur des pratiques culturelles de référence et de les faire travailler par des pratiques d'enseignement dans le sens

(47) F. SUBLET et Y. PRETEUR, "Le rapport à l'objet-livre dans le développement des compétences de lecture au CM1-CM2 ", J.-M. Privat et Y. Reuter, op.cit., pp. 75-98. Les auteurs concluent ainsi leur étude : "Notre recherche a donc permis d'observer des relations fortes entre compétences générales de lecture, compétences relatives aux livres et types de pratiques et attitudes de lecture ". 
d'un accomplissement ou « d'un dépassement positif » (48) ou critique. Le maître joue bien alors son rôle de médiateur.

\section{4- Le travail d'institution des lecteurs est fondamentalement légiti- miste. A plusieurs titres.}

Le légitimisme concerne d'abord, à l'évidence le corpus étudié ou fréquenté.

Lire est ainsi implicitement synonyme de livre et livre est lui-même synonyme de livre de fiction, de romans en général. Non seulement le fictionnel est donc valorisé au détriment des lectures fonctionnelles ou documentaires mais à l'intérieur même des lectures de fiction, c'est le " littéraire » qui s'impose largement à l'exclusion des textes paralittéraires. Ainsi, au collège, a-t-on «le sentiment d'un enseignement du français bien campé sur des valeurs de tradition : des auteurs éprouvés, une certaine imperméabilité à la littérature étrangère ou au texte non-littéraire, une tolérance limitée à la littérature de jeunesse » (49). Au lycée, le corpus littéraire est lui aussi « extrêmement précis et délimité ", avec une une très grande "focalisation » sur quelques auteurs classiques et parmi leurs œuvres, une extraordinaire «polarisation » sur quelques scènes réputées d'anthologie (50). On comprend qu'au delà des supports et des genres de lecture sélectionnés, ce sont des modes, des enjeux et des projets de lecture sélectifs qui s'imposent ainsi en toute légitimité (et qui « marquent » toute lecture au-delà même de l'école) (51).

Le légitimisme concerne en second lieu, et plus essentiellement, la problématique générale du fonctionnement du champ. La théorie du champ culturel ou la logique de la production restreinte et de la production élargie et ses effets dans la pratique lecturale (ou scripturale (52)) sont, de fait, complètement ignorées ou passées sous silence (53). C'est à la fois regrettable et dangereux.

L'enseignant (et plus généralement le médiateur culturel) a tout intérêt pourtant à se situer sociologiquement dans le champ des pratiques de lecture :

(48) J.-F. HALTÉ, La didactique du français, Paris, P.U.F., coll. Que sais-je ?, 1992, p. 99.

(49) D. MANESSE, "Quels textes enseigne-t-on au collège d'aujourd'hui ? ", Le Français aujourd'hui, décembre 1992, № 100, pp. 47-55.

(50) A.R. De BEAUDRAP et B. VECK, “Bibliothèque implicite », Le Français aujourd'hui, juin 1993, № 102, pp. $75-76$.

Les auteurs rappellent que les manuels entérinent ces choix et précisent que cette sursélection est " un phénomène propre à la pratique enseignante puisqu'il manifeste des effets de rétrécissement par rapport aux I.O." ".

(51) M.-P. SCHMITT auteur d'une thèse sur « la formation des goûts littéraires dans l'enseignement secondaire ", conclut une courte étude sur La lecture des adolescents (Universalia, 1993, La politique, les connaissances, la culture en 1992, Encyclopaedia Universalis, Paris, pp. 295-298) en ces termes : "Les fondements du goût littéraire sont donc scolaires : ils informent toutes les lectures, même celles dans lesquelles ils semblent le plus farouchement niés» (allusion aux lectures «libres» des lycéens).

(52) Les publications pour la jeunesse (même celles réputées de qualité) proposent la même vision enchantée (idéaliste et stéréotypée) de la production littéraire : «Le 2 novembre 1857, Alphonse Daudet arrive à Paris. Sans le sous, avec quelques dictionnaires pour tout bagage! Mais son frère Ernest est là pour l'aider; pendant des mois, ils vont partager la même mansarde. Alphonse désire être écrivain et travaille avec acharnement ; il trouve même un éditeur pour ses poèmes ! II n'en revient pas [...]. Alphonse est maintenant reçu dans les salons parisiens. Comme ailleurs, sa beauté séduit les femmes, son intelligence attire les hommes. II devient le secrétaire du Duc de Morny. Son travail lui permet de continuer à écrire, il est enchanté " (Je bouquine, mai 1993, № 111, p. 91)

(53) Voir par exemple le № 32 de Pratiques, "La littérature et ses institutions » et le Manuel d'histoire littéraire de D. DUPONT, Y. REUTER et J.-M. ROSIER, Bruxelles-Paris, De Boeck- Duculot, 1, 1988.

II va sans dire que le travail sur la culture du livre et du lecteur est, malgré les directives officielles, massivement délaissé par les autres professeurs qui abandonnent ce travail pourtant « tranversal »aux seuls professeurs de français et aux documentalistes. Une pratique pluridisciplinaire des ouvrages techniques ou de vulgarisation scientifique, des documentaires sur les sujets les plus divers et des journaux ou magazines spécialisés ou non permettrait d'élargir et le champ des savoirs concernés par le livre et le cercle des lecteurs potentiels. 
d'une part il évitera l'imposition brutale ou maladroite de modèles arbitraires mais vécus comme évidents et évidemment désirables (violence symbolique), d'autre part il évaluera mieux et plus positivement la distance d'un certain nombre d'élèves aux valeurs de l'institution scolaire et donc les cheminements didactiques à imaginer en s'appuyant sur la logique propre de leurs pratiques (ni fatalisme sociologique, ni pathologisation des comportements culturellement non conformes ou déviants).

La prise en compte des lectorats comme l'une des instances constitutives du champ lectural pourrait sembler aller de soi. Or, on s'était plutôt contenté jusqu'ici de réfléchir sur les structures de (re)production des biens culturels au détriment d'une analyse fine et sérieuse des structures de leur consommation. Autrement dit, mettre l'accent sur le champ du lecteur présente à la fois un enjeu théorique (ne pas penser la production sans la réception et ses effets de retour), politique (le retour de l'acteur et son efficace spécifique dans le cadre de macro-contraintes) et didactique (connaître les places culturelles de chacun pour ne pas faire des propositions " déplacées "). Le tableau qui suit vise à formaliser (et à schématiser) selon un modéle binaire la structure fondamentale du champ de la consommation lecturale, homologue au champ de la production (54). Ce tableau va être commenté dans les pages qui suivent.

Les différents traits pertinents qui composent ce système lectural sont dans une relation structurale d'oppositions et de solidarités qui les rend à la fois culturellement nécessaires et arbitraires, c'est-à-dire pris dans un jeu incessant de tensions distinctives et évolutives. Mais il ne faut pas perdre de vue non plus que les paradigmes " consommation cultivée » et " grande consommation " constituent eux-mêmes des sous-systèmes très cohérents qui ont une très forte logique interne.

On peut même estimer que le système de schèmes de pensée, de discours et de pratiques des divers lectorats est pour une bonne part le produit de l'intériorisation de ces oppositions constitutives de la structure du champ. Autrement dit, «ce qui est pertinent n'est pas une position en soi mais le système des oppositions entre positions différentes caractérisables par leur définition institutionnelle et par les propriétés de trajectoire des agents qui les occupent » (55).

1.- Le lecteur lettré (du moins le modèle idéal qu'en proposent les instances les plus légitimes) ne recherche pas directement dans l'acte même de la lecture, une quelconque rentabilité économique ou sociale. Cette préoccupation impure viendrait pervertir la délectation purement esthétique des œuvres légitimes. $\mathrm{Ce}$ mode d'appréhension des œuvres a bien sûr partie liée avec « l'autonomisation du champ de production littéraire et l'apparition corrélative d'œuvres demandant à être lues (et relues) en elles-mêmes et pour elles-mêmes » (56).

II va de soi que pour ce type de lectorat la valeur marchande des livres n'est pas un critère explicitement pris en compte (il n'est pas de bon goût de demander le prix d'un ouvrage par exemple et les émissions les plus "littéraires » n'en parlent jamais). L'économie de cette logique est celle d'un monde économique renversé où les profits symboliques sont à la mesure de la distance prise à

(54) On se reportera à l'article d'Yves REUTER (Pratiques, № 32, "La littérature et ses institutions ", pp. 5-30 ou au Manuel, op.cit., chapitres I et II notamment.

(55) L. PINTO, "Expérience vécue et exigence scientifique d'objectivité ", P. CHAMPAGNE, R. LENOIR, D. MERLLIÉ, L. PINTO, Initiation à la pratique sociologique, Paris, Dunod, 1990, p. 27.

(56) P. BOURDIEU, Les Règles de l'art, pp. 417-418. 


\section{Champ du lecteur}

\begin{tabular}{|c|c|}
\hline $\begin{array}{l}\text { Consommation cultivée } \\
\text { Lectorat lettré }\end{array}$ & $\begin{array}{l}\text { Grande consommation } \\
\text { Grand public }\end{array}$ \\
\hline \multicolumn{2}{|l|}{ 1- Fonctions } \\
\hline $\begin{array}{l}\text { dénégation de l'économie } \\
\text { recherche d'un capital culturel } \\
\text { et symbolique } \\
\text { intérêt désintéressé } \\
\text { lecture «cultivée " }\end{array}$ & $\begin{array}{l}\text { neutralisation des enjeux culturels } \\
\text { recherche d'une valeur pratique } \\
\text { désintérêt intéressé } \\
\text { lecture fonctionnelle }\end{array}$ \\
\hline \multicolumn{2}{|l|}{ 2- Logiques de consommation } \\
\hline $\begin{array}{l}\text { résistance à toute forme appuyée de } \\
\text { lecture dirigée } \\
\text { sélection distinctive } \\
\text { pacte artistique }\end{array}$ & $\begin{array}{l}\text { perception de l'offre sélectionnée } \\
\text { primat au livre consensuel } \\
\text { pacte référentiel }\end{array}$ \\
\hline \multicolumn{2}{|l|}{ 3- Modalités de lecture } \\
\hline $\begin{array}{l}\text { cycle de consommation continu } \\
\text { acceptation du risque d'une écriture } \\
\text { lecture comme « travail » } \\
\text { lecture inclusive }\end{array}$ & $\begin{array}{l}\text { cycle de consommation discontinu } \\
\text { quête de gratifications assurées } \\
\text { lecture comme « repos» } \\
\text { lecture exclusive }\end{array}$ \\
\hline \multicolumn{2}{|l|}{ 4- Styles d'appropriation } \\
\hline $\begin{array}{l}\text { lecture créative et critique } \\
\text { multiples réseaux d'approvisionnement } \\
\text { lecture intensive }\end{array}$ & $\begin{array}{l}\text { lecture d'affiliation } \\
\text { réseaux d'approvisionnement } \\
\text { peu diversifiés } \\
\text { lecture extensive }\end{array}$ \\
\hline \multicolumn{2}{|l|}{$\begin{array}{l}\text { 5- Rapports à la pratique } \\
\text { professionnelle }\end{array}$} \\
\hline $\begin{array}{l}\text { indifférenciation relative, lectures } \\
\text { professionnelles et personnelles } \\
\text { profits durables } \\
\text { dispose de plusieurs "marchés } \\
\text { de lecture " }\end{array}$ & $\begin{array}{l}\text { profession vs lecture de loisir } \\
\text { profits fugaces } \\
\text { "marché de lecture " restreint }\end{array}$ \\
\hline
\end{tabular}

\section{6- Discours sur " les autres "} et représentations
misérabilisme
élitisme
populisme
poujadisme 
l'égard des nécessités et contraintes réputées externes à l'œuvre et à sa consommation. L'investissement cultivé exclut donc que l'on s'investisse dans un calcul d'investissement et que l'on recherche autre chose qu'un profit culturel et symbolique, excluant ou discréditant, ce faisant, la recherche de toute autre forme de profit.

Ce qui est visé c'est la recherche d'une culture de qualité, à la fois reconnue et personnelle ; la conversion de cette capitalisation culturelle en termes de places et de postes socialement et économiquement intéressants est une éventualité acceptée (à long terme) mais non ostensiblement revendiquée ni même exigée.

Ce rapport aristocratique ou romantique à la lecture pure et gratuite, cet intérêt désintéressé qui n'a pas à se justifier et trouve sa propre raison ou sa propre fin dans son exercice même (et dans les jouissances subtilement distinctives qu'elle peut procurer), a évidemment pour objet d'élection privilégié la Littérature, ou même plus exactement la lecture littéraire des textes littéraires, « célébration sacralisante des classiques [...] ou ronron sacerdotal de la tradition scolaire » (57). C'est dire si l'institution du lecteur cultivé concerne aussi bien " la délimitation du corpus des textes consacrés par l'institution que la définition du mode de lecture légitime qui appréhende selon des grilles plus ou moins codifiées des textes constitués en tant que réalités auto-suffisantes, enfermant en elles-mêmes leur raison d'être » (58).

Inversement, il paraîtrait incongru au grand public de considérer ses propres lectures comme "culturelles "; nombre d'enquêtes ethnologiques montrent au contraire qu'il s'en défend : « Je lis pour mon plaisir, ce que j'ai envie, je n'ai pas de prétentions intellectuelles vous savez " ; "Je lis comme çà, quand j'ai un moment » ; « Non, je n'ai pas de projet de lectures, je lis ce qui me tombe sous la main, au petit bonheur » ; "Pour moi, c'est pas de la culture, c'est simplement pour me divertir, même si parfois çà peut me cultiver, si on peut dire ". Les lectures occupent les «temps morts " de la vie personnelle ou sociale (trajets, attentes, pauses, hospitalisation) et concernent un temps résiduel où on lit faute de pouvoir faire autre chose, faute de mieux.

La lecture est une activité fonctionnelle au sens où elle correspond a un besoin pratique (goût pour les livres pratiques, les encyclopédies, les dictionnaires, les informations locales ou régionales) ou une activité fusionnelle au sens où elle correspond a un besoin psychologique, socialement conditionné, d'oublier le réel pénible ou monotone et de partager, un moment au moins, le destin de héros hors du commun : "Ces livres qui s'adressent au plus grand nombre devraient nous réchauffer le cœur, nous donner espoir, nous émouvoir enfin "; "Toute vêtue de rose, Barbara Cartland ne poursuit qu'un objectif : donner du bonheur avec de belles histoires d'amour »; "Quoi de plus savoureux qu'un mélodrame de qualité, avec son lots d'émotion et de rebondissements ? ". La lecture est alors un service ou un pis aller, ce lectorat étant en général suffisamment conscient de la vanité sociale et de l'inutilité professionnelle d'une pratique concertée et soutenue de lecture mais aussi de son incapacité culturelle à pratiquer une lecture légitime. II y a donc un désintérêt intéressé dans ces stratégies d'évitement qui permettent 
de se soustraire à la confrontation avec les verdicts déclassants de la culture cultivée ou savante (59).

2.- Le lectorat lettré aime être libre de ses choix, ou du moins de choisir de façon très raisonnée et privée ses sources d'information (conversations avec des amis choisis, lectures de critiques spécialisés, consultations de travaux savants) ; la lettre de présentation du Corpus de l'Encyclopaedia Universalis n'argumente pas autrement en proposant « 15 jours pour vous faire un jugement personnel sur son contenu »; ce lectorat répugne par dessus tout à se laisser imposer des lectures trop communément vantées (prix Goncourt par exemple) ou à lire comme tout le monde ce que tout le monde lit. II sélectionne ses lectures en fonction de critères distingués et distinctifs (telle édition ou telle collection ou telle traduction plutôt que telle autre ; tel type de roman avant-gardiste ou ascétiquement classique) et suspecte le succès : "Les premiers Duras, oui, c'est vrai, mais maintenant... ».

Le pacte de lecture (littéraire) l'amène à être sensible enfin à la manière, au style, à la forme, à mettre à distance le texte dans un discours critique ou dans une tentative de re-création.

A l'opposé, le lectorat élargi privilégie l'offre sélectionnée par les grands intermédiaires médiatiques de lecture: il reconnaît la valeur au succès («Adapté à la télé » est un excellent argument de vente). II est ainsi concerné par les diverses formules de clubs du livre et par la publicité du livre dans la presse écrite ou audio-visuelle. Cette attention relative à l'information littéraire grand public repose sur une double logique : d'une part, l'investissement dans la lecture est précaire et peu valorisé (ce qui rend improbable et "coûteux ", disproportionnée, toute démarche d'accés à des informations compliquées, spécialisées ou réservées), d'autre part, l'insécurité culturelle de ce lectorat est telle qu'il est toujours ressenti comme risqué de s'aventurer dans des lectures qu'un large consensus culturel ne cautionne pas. Ce serait dépenser du temps et de l'argent pour un livre peut-être décevant ou illisible alors que ce qui est prioritairement attendu de la lecture c'est un service pratique et précis ou une distraction à coup sûr, bref, un usage garanti par le sens commun. D'où ces stratégies qui visent à maximiser les chances de profits et à minimiser les risques.

Ce même lecteur « joue le jeu » du texte romanesque, volontiers complice du point de vue du narrateur, sensible aux péripéties et participant aux émotions soulignées qu'éprouvent des héros «bien vivant ». Cet acceptation du simulacre (discursif) pour équivalent de la réalité (référentielle), cet abandon " naïf » aux séductions de l'histoire trouve sa raison principale certes dans l'incapacité à produire des discours critiques organisés mais surtout dans la recherche, à travers la lecture, simplement, d'un enchantement momentané ou du moins " dont les effets sensibles ne vont guère au-delà du temps consacré à la consommation » (60).

(59) Pour une analyse plus nuancée des pratiques des lectorats populaires (lectorats ouvriers, lectorats militants, autodidactes, etc.), voir entre autres M. NAFFRÉCHOUX, «Des lecteurs qui s'ignorent, les formes populaires de la lecture ", Bulletin des Bibliothèques de France, t. 32, № 5, 1987 ; N. ROBINE, op. cit., et les remarquables travaux, historiques ou ethnologiques de A.-M. THIESSE, Le roman au quotidien, lecteurs et lectures populaires à la Belle Epoque, Paris, Le Chemin Vert, 1984 et de J. BAHLOUL, Lectures précaires, études sociologiques sur les faibles lecteurs, Paris, Service des études et de la recherche, Bibliothèque publique d'information, Centre Georges Pompidou, 1988.

(60) CI. LAFARGE, "Usages des fictions ", La valeur littéraire, figuration littéraire et usages sociaux des fictions, Paris, Fayard, 1983, pp. 209-282. 
3.- La consommation cultivée est le plus souvent portée par un projet lectural et plus généralement culturel qui engage des programmes de lectures; ces programmes sont d'ailleurs plus ou moins respectés car en matière de culture comme dans d'autres domaines, le privilège des privilèges consiste à prendre des libertés avec ses propres règles. A la différence du petit porteur culturel ou de l'austère autodidacte, le lettré doit pouvoir en effet manifester sa maîtrise du jeu et des enjeux culturels en saisissant une superbe occasion de lecture lorsqu'elle se présente. II n'est pas dépendant d'une offre calibrée et planifiée par d'autres mais organise lui-même la bibliothèque de ses lectures.

Il se risque volontiers - c'est en fait un devoir statutaire - vers des œuvres nouvelles, inédites, à diffusion restreinte ou confidentielle, et accepte de partager l'aventure d'une écriture. II est vrai que pour lui relire et annoter ses lectures fait partie du contrat culturel de lecture dans la mesure où lire est accepté comme un travail intellectuel, parfois éprouvant. Un livre trop « facile », qui ne suppose pas une certaine concentration, en un mot qui ne résiste pas assez, n'est-il pas assez systématiquement considéré comme un mauvais livre? Le lecteur lettré peut, comme Flaubert, sortir « fourbu » d'une lecture.

On sait enfin que les grands lecteurs et les lecteurs les plus légitimes sont à la fois très sélectifs dans leurs lectures mais dans le même temps raisonnablement éclectiques. Les enquêtes statistiques confirment toutes qu'une des caractéristiques majeures de ce lectorat est sa propension à multiplier ses choix de lecture, à être ouvert à la variété de l'offre (à l'intérieur du bon goût culturel, dont les frontières sont cependant fort labiles puisque par exemple le feuilleton frénétiquement populaire d'hier peut devenir aujourd'hui l'objet d'une lecture savante, au deuxième degré, etc.).

Il est par contre dans la logique de la grande consommation de lire de façon fragmentée, discontinue, dans les interstices que laissent la vie quotidienne ou les moments de loisirs. La lecteur ne s'accorde pas facilement un temps spécifique pour lire et n'a pas non plus forcément une bibliothèque pour ranger des livres qu'un certain hasard rassemble. La lecture a quelque chose d'aléatoire ou parfois, inversement, de routinisée (presse régionale, romans sentimentaux, magazines). C'est un renseignement, un savoir précis sur le monde ou une gratification émotionnelle qui est recherché: «Les Harlequins, c'est comme les hommes, c'est difficile de s'endormir sans ", assure la publicité.

Le bénéfice de la lecture doit être à la fois concret et non différé. N. Robine a montré par exemple combien cette disposition du lecteur grand public le conduisait à rechercher des romans où l'on est plongé dés le début dans une action qui se déroule linéairement, sans inutile complication d'écriture. L'intérêt aux documents qui donnent des réponses simples et vivantes à des questions qui se posent dans la pratique et pour la pratique (la valorisation du dictionnaire illustré est l'exemple type) obéit à la même logique.

La lecture doit être utile ou distractive. C'est cet enjeu pragmatique qui explique que le texte ne doit pas poser de problème de traitement (caractères trop petits, mise en page sophistiquée, etc.) ou d'interprétation trop ambiguë ou trop complexe. Le lecteur aime ainsi suivre l'écriture d'une aventure et non se perdre dans des problèmes d'élaboration du sens. Si cette lecture d'évasion ou de loisir doit être aisée pour rendre le service que l'on attend d'elle (les grandes surfaces de distribution signalent souvent l'emplacement des livres par le panneau «Loisirs...Livres »), alors il va de soi que les constructions stéréoty- 
pées correspondent parfaitement à cette attente d'un plaisir assuré et facilement accessible. C'est souvent parce qu'il est « fourbu » que ce lectorat lit.

La grande consommation enfin, n'est pas délibérément variée. Le grand public valorise quelques genres et quelques fonctions de lecture, et s'y tient. On lit pour s'informer ou pour se distraire des choses connues, reconnues et abordables, à tous les sens du terme. On ne s'aventure guère à pratiquer des genres méconnus et à se confronter à des textes redoutables. La situation la plus conventionnelle est bien la lecture de la presse quotidienne régionale par l'homme, celle de romans sentimentaux par la femme (les librairies de grandes surfaces parlent d'ailleurs de "romans féminins »).

4.- Par définition, le lettré s'adonne à une lecture savante, imaginative, inventive, rebelle, intensément personnelle alors que le lecteur ordinaire reproduit les lectures convenues, apprises. Soit le moi du lettré se fond et se confond avec le moi créateur de l'auteur, soit il se livre à une lecture critique. Dans l'un ou l'autre cas, il se considère comme averti, dégagé des lectures dominantes qu'il tient souvent pour réductrices ou aliénantes.

De façon formelle (thèse, commentaires critiques, livres) ou informelle (conversations amicales, allusions critiques), le lecteur lettré recherche le reconnaissance de ses pairs, à moins qu'il ne satisfasse d'un dialogue herméneutique narcissique. II est néanmoins toujours en relation avec un ensemble riche et varié de réseaux d'approvisionnement puisque la loi du cumul culturel joue là aussi : il cumule volontiers inscription en bibliothèque, abonnement à des revues, achats d'ouvrages dans des lieux divers, prêts amicaux de bouquins, livres offerts en différentes occasions.

Il est logique que dans ces conditions ce lectorat pratique une lecture intensive, c'est-à-dire à la fois très soutenue et à haut rendement culturel et social.

A l'inverse, le grand public lit sur un mode extensif, c'est-à-dire de façon périodique et avec un faible rendement culturel et social. D'ailleurs, son réseau d'approvisionnement est limité et en règle générale distant des lieux et modalités les plus légitimes d'offre du livre.

Les ambitions de ce lectorat ne sont pas d'ordre culturel et distinctif, au contraire. On lit ce qui se lit, ce que tout le monde lit, et on s'abandonne à une lecture conventionnelle. II ne s'agit pas de se singulariser, d'être original mais bien de partager des lectures communes sur un mode communément admis, des lectures qui rapprochent ou du moins qui donnent le sentiment de partager une culture commune au groupe auquel on se sent lié et relié.

5.- Le lecteur lettré est un professionnel de la lecture au sens où il peut en faire sa profession (professeurs, libraires, bibliothécaires) et parce que, comme dans les couches dominantes de façon générale, il n'y pas vraiment solution de continuité entre le temps de travail proprement dit et le temps personnel. II est ainsi difficile de dire s'il lit pour son plaisir ou pour son travail dans la mesure où l'espace professionnel recoupe partiellement l'espace privé et où une lecture personnelle à toute chance de servir tôt ou tard dans l'exercice du métier ; ainsi peut-il trouver un intérêt profond et profondément personnel à une lecture que son métier lui impose. Les enjeux et les profits de lecture sont durables (retraduits sous forme de diplômes, de notoriété ou d'avancement) et les occasions de parler spontanément ou dans un cadre institué de ses lectures sont nombreuses.

Le lecteur ordinaire, lui, n'a pas à proprement parler de lecture professionnelle 
(son poste de travail ne l'exige pas) et son désir est de s'installer chez lui avec un bon bouquin qui, précisément, lui fera oublier un moment la réalité qu'il vient de quitter. II en attend un plaisir momentané et ne dispose que rarement d'un public, même intime, pour évoquer ses lectures. II serait même souvent mal vu d'amener la conversation sur ce sujet.

6.- On comprend que cet ensemble d'oppositions dans la pratique lecturale crée les conditions de développement de discours affrontés, à la limite de l'affront. Les figures théoriques de cet affrontement relèvent d'une double dérive, la dérive légitimiste et la dérive relativiste. Dans un cas, le misérabilisme consistera à mépriser (de l'agression culturelle au déni de lecture) les lectures des "petits " lecteurs, au nom de la Culture et dans la méconnaissance de logiques cultures différentes ou antagonistes (" Harlequin, c'est incroyablement nul » ou "C'est le canard du coin, un vrai torchon ») ; dans l'autre cas, le populisme verra dans toute lecture «populaire » une manifestation d'une culture autonome et authentique dans l'oubli total des rapport de domination, de dépendance culturelles et de ses effets sur les pratiques (61). Inversement, le grand public peut soit reconnaître admirativement la légitime noblesse de la Culture (légitimisme élitiste des dominés), soit dénoncer agressivement les prétentions des prétendues «têtes » ("Deux intellectuels assis vont moins loin qu'un con qui marche... », dans la version médiatico-poujadiste de Michel Sardou).

Cette analyse différentielle des modes de socialisation culturelle et lectorale permet de comprendre pourquoi le conflit culturel est parfois inévitable et quels sont les problèmes de compromis ou d'ajustements qui se posent à la fois aux maîtres et à une fraction non négligeable d'élèves engagés dans les processus contradictoires de socialisation culturelle familiale et de socialisation culturelle scolaire. Un des effets redoutables du contact moyen (scolaire ?) avec la littérature notamment, n'est-il pas de " détruire l'expérience populaire » ou personnelle et de "laisser les gens formidablement démunis, c'est-à-dire entre deux culture, entre une culture originaire abolie et une culture savante qu'on a assez fréquentée pour ne plus pouvoir parler de la pluie et du beau temps, pour savoir tout ce qu'il ne faut pas dire, sans avoir plus rien d'autre à dire » (62).

II ne s'agit en effet ni de convertir à la culture consacrée, porté par un ardent prosélytisme, ni de réhabiliter le « populaire », poussé par un relativisme militant, mais d'aider à construire un parcours lectural : bref, s'éloigner du culte du livre et des seuls livres de Culture pour familiariser à la culture du livre et des livres par un travail critique et « expérienciel » d'appropriation culturelle des conditions culturelles d'accés et de pratique (ce qui n'est donc pas synonyme de réconduire le rapport lettré aux biens culturels ni laisser jouer, d'un autre côté, le rapport spectacularisé aux œuvres et aux producteurs starisées) (63).

(61) CI. GRIGNON et J.-Cl. PASSERON, Le savant et le populaire, misérabilisme et populisme en sociologie et en littérature, Paris, Hautes Etudes, Gallimard, Le Seuil, 1989.

(62) P. BOURDIEU, «La lecture, une pratique culturelle », Pratiques de la lecture, Marseille, Rivages, 1985, p. 228. Nous travaillons actuellement à comprendre comment l'Ecole «fabrique " de la " culture moyenne " et quels effets produit sur leurs discours et leurs pratiques le positionnement des enseignants, à mi-chemin en général (par fonction professionnelle et / ou trajectoire personnelle) de la répulsion pour la culture grand public et de la fascination pour la culture cultivée. Je me suis contenté ici de baliser le champ, sans décrire dans le détail les conséquences pédagogiques de cette double "proximité distante " (mise à distance et tenue à distance), sans préciser les tensions culturelles dues à cette relation de "voisinage structural " (P. BOURDIEU, La distinction, critique sociale du jugement, Ed. de Minuit, Paris, 1979, p. 64).

(63) O. DONNAT, « Démocratisation culturelle : la fin d'un mythe », Esprit, 3-4, 1991, p. 79. 
Ainsi, entre l'inculcation des valeurs patrimoniales (64) et l'idéologie de la lecture comme activité désirante, entre l'imposition culturelle et l'animation culturelle, il y a précisément la construction des médiations culturelles (65) qui s'efforce - comme on lira dans l'article de Dominique Lelièvre-Portalier et Marie-Christine Vinson sur la bouquinerie au collège - de tenir compte à la fois des effets de la domination culturelle et de la réalité de pratiques différentes ou divergentes (66).

\section{5o- Le travail d'institution des lecteurs est enfin beaucoup trop décon- textualisé et désocialisé.}

Tout semble fait, construit, organisé dans les situations scolaires de travail, pour que le lecteur se retrouve seul devant un objet textuel, dans un solitaire tête-à-texte (quand ce n'est pas un solennel tête-à-test !), et pour qu'il soit seulement une machine cognitive plus ou moins performante. Ce dispositif renvoie à la confusion qui régne dans l'imaginaire lettré moderne entre l'intimisation de l'acte de lecture proprement dit (acte privé et personnel, souvent) et les dimensions socialisées et socialisantes des pratiques de lecteurs. Or, "l'initiative de la lecture, la réception et la circulation des livres [...] s'inscrivent dans la complexité des interactions et des échanges qui composent la socialisation du lecteur » (67).

II y a mise entre parenthèse scolaire de la pratique, des conditions concrètes et des enjeux diversifiées de la pratique lecturale, neutralisation des fonctions impliquées ordinairement dans l'usage ordinaire ou le plus fréquent, savant ou non, du livre (68).

Le livre est réduit à sa lettre, lettre morte ou éternelle, et il est saisi comme un objet d'analyse, comme un bien symbolique sans fin pratique ou plus exactement qui est à soi sa propre fin, sans autre enjeu que celui d'être interprété. Ainsi s'accomplit l'autonomisation des lectures par rapport à leurs conditions sociales ou culturelles d'usage, en homologie à l'autonomisation relative du champ de la production culturelle et en prolongement de l'expérience bourgeoise du rapport au monde.

Cette culture livresque du livre, cette conception scolastique du lecteur (on pense aux célèbres analyses de Durkheim sur le formalisme esthético-littéraire), c'est la voie ouverte aux théories et aux pratiques qui font comme si la maîtrise théorique du code culturel suffisait à conférer la maîtrise pratique des usages socialement et culturellement appropriés.

II y a ainsi une véritable déréalisation du monde du lecteur sur ce marché protégé et assuré qu'est l'Ecole. On peut parler d'une atrophie de l'économie des pratiques lecturales et donc des conditions de l'apprentissage ; il faudrait refaire sur l'économie des pratiques lecturales les analyses que Bourdieu a

(64) Nous partageons les vues de M. WERNER et J.-M SCHAEFFER qui souhaitent que la littérature soit considérée comme " une pratique culturelle et non pas comme un patrimoine à reproduire dans l'enseignement " («Pourquoi l'histoire littéraire n'a-t-elle jamais réussi en France à se construire en discipline scientifique autonome? ", Le Monde, spécial salon du livre, 18 mars 1993, p. XII.

(65) J.-M. PRIVAT et Y. REUTER (ed.), Lectures et médiations culturelles, Lyon, P.U.L., 1991.

66) Sur les fondements théoriques de l'analyse idéologique et de l'analyse culturelle, voir C. GRIGNON et J.-C. PASSERON, op. cit., et sur leurs transpositions didactiques en termes de stratégies d'alternance et d'ambivalence, voir aussi J.-M. PRIVAT et Marie-Christine VINSON, « Les intermédiaires de lecture ", Pratiques, № 63, septembre 1989, pp. 63-101 et Y. REUTER, Pratiques, № 50, juin 1986, pp. 3-21.

(67) J. BAHLOUL, op. cit., pp. 18-19.

(68) Sur les différentes fonctions que les livres peuvent remplir " simultanément pour un même individu ", voir par exemple les remarques de F. de SINGLY, "La lecture de livres pendant la jeunesse : statut et fonction ", Lire en France aujourd'hui, sous la dir. de M. Poulain, Editions du Cercle de la Librairie, coll. Bibliothèques, Paris, 1993, pp. 137-162. 
données sur «l'économie des échanges linguistiques 》 (69) pour dépasser ici le « philologisme » qui met entre parenthèses les jeux et enjeux d'une parole et d'une performance, là ce que nous proposons d'appeler le "lecturisme ".

En un mot, on pourrait dire que l'Ecole s'efforce de développer une pratique culturelle sans... culture pratique !

Cette saisie intellectualiste et subjectiviste induit une conception strictement herméneutique de la lecture et une vision « abstraite » du monde culturel : il s'agit de « jouer sérieusement » et de faire preuve, dans le « commerce des livres » d'esprit et de finesse («vie intérieure et culture » disent les rapports de concours, "garder à l'enseignement des lettres son caractère de discipline désintéressée et culturelle », " développer le goût de la lecture et de la culture désintéressée » disent et répètent les instructions pédagogiques pour les classes de seconde et de première) (70). La version plus commune parle de «plaisir »...

On est loin de ces "marchés de lecture » dont parle encore Bourdieu (71) - " on lit quand on a un marché sur lequel on peut placer des discours sur la lecture »-, des interlectoralités et des sociabilités en général où l'on se lecture (on devrait dire « se lecturer » comme on dit justement « se cultiver », travail du sujet sur lui-même dans des réseaux d'interactions sociales et symboliques).

En effet, " même lorsqu'elle se confond avec ce tête-à-tête intime du lecteur avec son livre qui exige silence, calme, retrait du monde presque, la lecture est échange, partage social d'un message. Le choix d'un titre, la rencontre d'un texte, son achat ou son emprunt supposent déjà tout un réseau social complexe d'individus que la lecture, à des titres divers, rassemble dans des dispositifs plus ou moins élaborés ou institutionnalisés qui sont le théâtre de gestes, d'habitudes, de façons de dire ou de faire. De même, le livre lu, on en parle, on le prête, on le raconte ou le commente. D'autres rencontres s'organisent alors, spontanées ou réglées, qui prolongent le rapport premier du texte au lecteur, l'enrichissent et en définitive préparent d'autres lectures. Bref, la lecture implique une sociabilité large » (72).

La construction d'un habitus de lecture, cultivé ou non, n'est donc pas seulement " une opération abstraite d'intellection » mais aussi et toujours "pratique incarnée dans des gestes, des espaces, des habitudes »; mieux, c'est dans ces "communautés de lecteurs " (73) que se dit, se redit et se renégocie perpétuellement la croyance (ou non) en l'importance de la lecture, de telles lectures et de telles modalités de lecture. Autrement dit les sociabilités lectrices, formelles ou informelles, professionnelles, associatives, familiales, communautaires ou simplement amicales, participent de la production et de la reproduction de la nécessaire croyance dans l'intérêt d'une pratique : en ce sens elles constituent un moment essentiel dans la production sociale et culturelle du lecteur.

II serait donc précieux d'être attentif aux développements des réseaux de sociabilités car " on a d'autant plus de chance de lire que dans la zone de relations proches, on fréquente des gens qui vont prêter, conseiller, parler le

(69) P. BOURDIEU, “L'économie des échanges linguistiques ", Langue française, 34, mai 1977, pp. 17-34. II s'agit, dans la ligne des analyses de Bakhtine, de démontrer que ce qui est acquis est inséparablement " la maîtrise pratique du langage et la maîtrise pratique des situations " qui permettent aux compétences d'être mises en œuvre dans des situations réelles.

(70) Ministère de l'Education nationale et de la culture, Français, classe de seconde, première et terminale, horaires / objectifs / programmes / instructions, CNDP, réédition 1992, p. 55 et p. 66

(71) P. BOURDIEU, “ La lecture, une pratique culturelle », Pratiques de lecture, op. cit., p. 224

(72) J. HÉBRARD, "Les nouveaux lecteurs ", Histoire de l'édition française, (R. CHARTIER et H.J. MARTIN ed.), Paris, Promodis, 1985, tome 3, pp. 495-496.

(73) R. CHARTIER, L'Ordre des livres, Aix-en-Provence, Alinéa, 1992, "Communautés de lecteurs », pp. 13-33. 
livre » (74). Aussi peut-on dire que " toutes les initiatives dans les collèges, dans les lycées, dans les universités, hors des murs de l'école, qui font que la parole autour du livre dépasse le cercle des intimes sont bonnes » (75).

Les entreprises de vente du livre par correspondance ont bien compris ce rôle des sociabilités : France-Loisirs est "Le club où il fait bon lire " et tout un dispositif de relations socio-affectives (mimées) est proposé aux membres du club ; le parrainage en est la formule la plus manifeste.

\section{Conclusion}

L'Ecole serait-elle donc « la seule institution qui, emportée par l'urgence pédagogique des apprentissages à réaliser, manque en grande partie ses objectifs parce qu'elle ne se donne pas le temps de ménager une médiation entre l'enfant et la culture écrite ? ». Pourtant, l'école est capable d'inscrire "l'acculturation qu'elle propose dans des pratiques culturelles propres aux milieux sur lesquels elle travaille " à condition qu'une série de médiations soit trouvée et qu'elle " accepte de sortir des modèles stéréotypés qu'elle véhicule » (76). Le modèle présenté permettra peutêtre, on l'a dit, une analyse critique et constructive des divers et innombrables «projets lecture »; de plus, parce que le système ethno-didactique a sa cohérence interne (« ponctuel » et « partiel » se présupposent réciproquement, et ainsi de suite) sa traduction, même minimale, dans les faits, nous paraît plus importante qu'une surinvestissement sur un point particulier.

Reste que la didactisation généralisée (et ambitieuse) des pratiques de lecture que nous proposons pour des lecteurs concrets, nombreux et polymorphes suppose que les maîtres travaillent... à maîtriser autant que faire se peut leurs propres positions et prédispositions culturelles (77) et qu'ils œuvrent, pour mettre en place trois situations pédagogiques essentielles:

- le travail en équipe d'enseignants, de la maternelle aux lycées ;

- le travail en projet, pour que la formation culturelle s'intègre de façon dynamique et créative à la vie personnelle des élèves (le rôle des pairs mais aussi celui des parents est à prendre en compte) ;

- le travail en réseaux (rôle des centres documentaires et des bibliothèques scolaires, des médiathèques et bibliothèques publiques, des librairies et autres commerces du livre enfin), pour assurer la diversité et l'efficacité des apprentissages, par delà le cursus scolaire et (enfin ?) détachés de la présence « instituante » des prescripteurs institutionnels ou des médiateurs professionnels.

(74) J.-C. POMPOUGNAC, « La lecture publique entre discours et pratiques », Esprit, 3-4, 1991, p. 113.

(75) F. de SINGLY, Les jeunes et la lecture, Ministère de l'Education nationale et de la Culture, Les dossiers Education et Formations, 24, janvier 1993, p. 126.

(76) J. HÉBRARD, Ecole et alphabétisation au XIXe siècle, Annales E.S.C., janv-février 1980, pp. 75-76.

Le "Goncourt des Lycéens " (lire en quelques semaines et pour de vrai dix romans " goncourables ", parler de ses lectures et de ses découvertes, jour après jour, au hasard des rencontres amicales ou des échanges familiaux, rédiger des critiques publiées par la presse régionale ou affichées au CDI, attendre fébrilement l'accueil que réservent les journaux spécialisés aux divers romans, désigner le livre à primer, rencontrer des écrivains, des représentants de maison d'édition, des membres de jurys littéraires, etc.) est un exemple de cette nouvelle configuration didactique qui fait une large place au travail des sociabilités. Pour une analyse critique de cette expérience, voir M. BURGOS et J.-M. PRIVAT, "Le Goncourt des lycéens : vers une sociabilité littéraire ? ", Lire en France aujourd'hui, op.cit., pp. 163-182, et dans ce numéro la dernière partie de l'article de M. Burgos.

(77) Pour prolonger la réflexion, on pourra par exemple se reporter aux analyses de M. PINÇON et M. PINÇONCHARLOT, "Classes moyennes, enjeux culturels et trajectoires sociales ", Les Cahiers de l'Animation, 1985, № 53, pp. 102-111 ou à celles de J.-CI. PASSERON, Le Raisonnement sociologique, Paris, Nathan, Essais et Recherches, 1991, notamment les pages 309 et suivantes consacrées aux « acteurs de l'action culturelle ». 